\title{
A generalization of the alcove model and its applications
}

\author{
Cristian Lenart • Arthur Lubovsky
}

Received: 16 December 2013 / Accepted: 13 August 2014 / Published online: 26 August 2014

(C) Springer Science+Business Media New York 2014

\begin{abstract}
The alcove model of the first author and Postnikov uniformly describes highest weight crystals of semisimple Lie algebras. We construct a generalization, called the quantum alcove model. In joint work of the first author with Naito, Sagaki, Schilling, and Shimozono, this was shown to uniformly describe tensor products of column shape Kirillov-Reshetikhin crystals in all untwisted affine types; moreover, an efficient formula for the corresponding energy function is available. In the second part of this paper, we specialize the quantum alcove model to types $A$ and $C$. We give explicit affine crystal isomorphisms from the specialized quantum alcove model to the corresponding tensor products of column shape Kirillov-Reshetikhin crystals, which are realized in terms of Kashiwara-Nakashima columns.
\end{abstract}

Keywords Kirillov-Reshetikhin crystals - Energy function - Alcove model · Quantum Bruhat graph · Kashiwara-Nakashima columns

Mathematics Subject Classification Primary 05E10 - Secondary 20G42

\section{Introduction}

Kashiwara's crystals [11] are colored directed graphs encoding the structure of certain bases (called crystal bases) for certain representations of quantum groups $U_{q}(\mathfrak{g})$ as $q$ goes to zero. The first author and Postnikov [23,24] defined the so-called alcove

C. Lenart $(\varangle) \cdot$ A. Lubovsky

Department of Mathematics and Statistics, State University of New York at Albany, Albany,

NY 12222, USA

e-mail: clenart@albany.edu

A. Lubovsky

e-mail: alubovsky@albany.edu 
model for highest weight crystals associated to a semisimple Lie algebra $\mathfrak{g}$ (in fact, the model was defined more generally, for symmetrizable Kac-Moody algebras $\mathfrak{g}$ ). A related model is the one of Gaussent-Littelmann, based on LS-galleries [7]. Both models are discrete counterparts of the celebrated Littelmann path model [26,27].

In this paper, we construct a generalization of the alcove model, which we call the quantum alcove model, as it is based on enumerating paths in the so-called quantum Bruhat graph of the corresponding finite Weyl group. This graph originates in the quantum cohomology theory for flag varieties [6], and was first studied in [2]. The path enumeration is determined by the choice of a certain sequence of alcoves (an alcove path or, equivalently, a $\lambda$-chain of roots), like in the classical alcove model. If we restrict to paths in the Hasse diagram of the Bruhat order, we recover the classical alcove model. The mentioned paths in the quantum Bruhat graph first appeared in [18], where they index the terms in the specialization $t=0$ of the Ram-Yip formula [30] for Macdonald polynomials $P_{\lambda}(X ; q, t)$. We construct combinatorial crystal operators on the mentioned paths, and prove various properties of them.

The main application [21,22] is that the new model uniformly describes tensor products of column shape Kirillov-Reshetikhin (KR) crystals [13], for all untwisted affine types. (KR crystals correspond to certain finite-dimensional representations of affine algebras.) More precisely, the model realizes the crystal operators on the mentioned tensor product, and also gives an efficient formula (based on the so-called height statistic) for the corresponding energy function [8]. (The energy can be viewed as an affine grading on a tensor product of KR crystals [28,32].) This result, combined with the Ram-Yip formula for Macdonald polynomials [30], implies that the graded character of a tensor product of column shape KR modules (the grading being by the energy function) concides with the corresponding Macdonald polynomial specialized at $t=0$ [22].

In the second part of this paper, we specialize the quantum alcove model to types $A$ and $C$, and prove that the bijections constructed in [18], from the objects of the specialized quantum alcove model to the tensor products of the corresponding KashiwaraNakashima $(K N)$ columns [12], are affine crystal isomorphisms. (A column shape KR crystal is realized by a KN column in these cases.) Note that this result has no overlap with the type-independent result in $[21,22]$, because the $\lambda$-chains on which the quantum alcove model is based in the two cases are different. Moreover, note that having such explicit bijections to models based on diagram fillings, which are known to be crystal isomorphisms, is important for the following reason. It is often easier to define extra structure on the quantum alcove model, which describes additional structure of the crystal, and only then translate it to the models based on fillings via the bijection. One such example was mentioned above, in connection with the energy function; the height statistic in the quantum alcove model has been translated to the so-called charge statistic for fillings in types $A$ and $C$ in [18], while type $B$ is currently under investigation in [1]. Another example is related to the combinatorial $R$-matrix mentioned below.

We also conjecture that, like the alcove model, its generalization given here is independent of the $\lambda$-chain of roots on which the whole construction is based, cf. [15]. This conjecture is currently under investigation in [19]. We intend to realize an affine crystal isomorphism between the models based on two $\lambda$-chains by extending to the 
quantum alcove model the alcove model version of Schützenberger's jeu de taquin [5] on Young tableaux in [15]; the latter is based on so-called Yang-Baxter moves. Another application of this construction would be a uniform realization of the combinatorial $R$-matrix (i.e., the unique affine crystal isomorphism commuting factors in a tensor product of KR crystals).

\section{Background}

Let $\mathfrak{g}$ be a complex semisimple Lie algebra, and $\mathfrak{h}$ a Cartan subalgebra, whose rank is $r$. Let $\Phi \subset \mathfrak{h}^{*}$ be the corresponding irreducible root system, $\mathfrak{h}_{\mathbb{R}}^{*} \subset \mathfrak{h}$ the real span of the roots, and $\Phi^{+} \subset \Phi$ the set of positive roots. Let $\Phi^{-}:=\Phi \backslash \Phi^{+}$. For $\alpha \in \Phi$, we will say that $\alpha>0$ if $\alpha \in \Phi^{+}$, and $\alpha<0$ if $\alpha \in \Phi^{-}$. The sign of the root $\alpha$, denoted $\operatorname{sgn}(\alpha)$, is defined to be 1 if $\alpha \in \Phi^{+}$, and -1 otherwise. Let $|\alpha|=\operatorname{sgn}(\alpha) \alpha$. Let $\rho:=\frac{1}{2}\left(\sum_{\alpha \in \Phi^{+}} \alpha\right)$. Let $\alpha_{1}, \ldots, \alpha_{r} \in \Phi^{+}$be the corresponding simple roots, and $s_{i}:=s_{\alpha_{i}}$ the corresponding simple reflections. We denote $\langle\cdot, \cdot\rangle$ the nondegenerate scalar product on $\mathfrak{h}_{\mathbb{R}}^{*}$ induced by the Killing form. Given a root $\alpha$, we consider the corresponding coroot $\alpha^{\vee}:=2 \alpha /\langle\alpha, \alpha\rangle$ and reflection $s_{\alpha}$. If $\alpha=\sum_{i} c_{i} \alpha_{i}$, then the height of $\alpha$, denoted by ht $(\alpha)$, is given by ht $(\alpha):=\sum_{i} c_{i}$. We denote by $\widetilde{\alpha}$ the highest root in $\Phi^{+}$; we let $\theta=\alpha_{0}:=-\widetilde{\alpha}$ and $s_{0}:=s_{\widetilde{\alpha}}$.

Let $W$ be the corresponding Weyl group. The length function on $W$ is denoted by $\ell(\cdot)$. The Bruhat order on $W$ is defined by its covers $w \lessdot w s_{\alpha}$, for $\alpha \in \Phi^{+}$, if $\ell\left(w s_{\alpha}\right)=\ell(w)+1$. Define $w \triangleleft w s_{\alpha}$, for $\alpha \in \Phi^{+}$, if $\ell\left(w s_{\alpha}\right)=\ell(w)-2 \mathrm{ht}\left(\alpha^{\vee}\right)+1$. The quantum Bruhat graph [6] is the directed graph on $W$ with edges labeled by positive roots

$$
w \stackrel{\alpha}{\longrightarrow} w s_{\alpha} \quad \text { for } w \lessdot w s_{\alpha} \text { or } w \triangleleft w s_{\alpha} ;
$$

see Example 4.2.

The weight lattice $\Lambda$ is given by

$$
\Lambda:=\left\{\lambda \in \mathfrak{h}_{\mathbb{R}}^{*}:\left\langle\lambda, \alpha^{\vee}\right\rangle \in \mathbb{Z} \text { for any } \alpha \in \Phi\right\}
$$

The weight lattice $\Lambda$ is generated by the fundamental weights $\omega_{1}, \ldots \omega_{r}$, which form the dual basis to the basis of simple coroots, i.e., $\left\langle\omega_{i}, \alpha_{j}^{\vee}\right\rangle=\delta_{i j}$. The set $\Lambda^{+}$of dominant weights is given by

$$
\Lambda^{+}:=\left\{\lambda \in \Lambda:\left\langle\lambda, \alpha^{\vee}\right\rangle \geq 0 \text { for any } \alpha \in \Phi^{+}\right\} .
$$

Given $\alpha \in \Phi$ and $k \in \mathbb{Z}$, we denote by $s_{\alpha, k}$ the reflection in the affine hyperplane

$$
H_{\alpha, k}:=\left\{\lambda \in \mathfrak{h}_{\mathbb{R}}^{*}:\left\langle\lambda, \alpha^{\vee}\right\rangle=k\right\}
$$

These reflections generate the affine Weyl group $W_{\text {aff }}$ for the dual root system $\Phi^{\vee}:=$ $\left\{\alpha^{\vee} \mid \alpha \in \Phi\right\}$. The hyperplanes $H_{\alpha, k}$ divide the real vector space $\mathfrak{h}_{\mathbb{R}}^{*}$ into open regions, called alcoves. The fundamental alcove $A_{\circ}$ is given by

$$
A_{\circ}:=\left\{\lambda \in \mathfrak{h}_{\mathbb{R}}^{*} \mid 0<\left\langle\lambda, \alpha^{\vee}\right\rangle<1 \text { for all } \alpha \in \Phi^{+}\right\} .
$$


We will need the following properties of the quantum Bruhat graph which were proved in [21]; more precisely, Lemma 2.1 below is a simplified version of Proposition 5.4.2 in the mentioned paper, whereas Lemmas 2.2 and 2.3 below are simplified versions of different parts of the Diamond Lemma 5.5.2.

Lemma 2.1 Let $w \in W$. We have $w^{-1}(\theta)>0$ if and only if $w \triangleleft s_{\theta} w$. We also have $w^{-1}(\theta)<0$ if and only if $s_{\theta} w \triangleleft w$.

Lemma 2.2 Let $w \in W$, let $\alpha$ be a simple root, $\beta \in \Phi^{+}$, and assume $s_{\alpha} w \neq w s_{\beta}$. Then $w \lessdot s_{\alpha} w$ and $w \longrightarrow w s_{\beta}$ if and only if $w s_{\beta} \lessdot s_{\alpha} w s_{\beta}$ and $s_{\alpha} w \longrightarrow s_{\alpha} w s_{\beta}$, cf. the diagram below. Furthermore, in this context we have $w \lessdot w s_{\beta}$ if and only if $s_{\alpha} w \lessdot s_{\alpha} w s_{\beta}$.

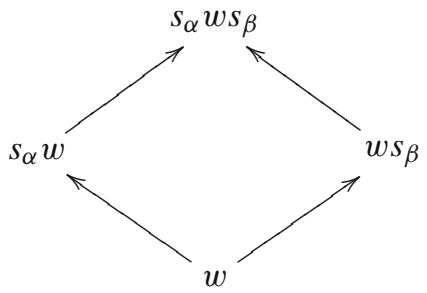

Lemma 2.3 Let $w \in W, \beta \in \Phi^{+}$, and assume $s_{\theta} w \neq w s_{\beta}$. Then $w \triangleleft s_{\theta} w$ and $w \longrightarrow w s_{\beta}$ if and only if $w s_{\beta} \triangleleft s_{\theta} w s_{\beta}$ and $s_{\theta} w \longrightarrow s_{\theta} w s_{\beta}$.

\subsection{Kirillov-Reshetikhin (KR) crystals}

A $\mathfrak{g}$-crystal (for a symmetrizable Kac-Moody $\mathfrak{g}$ ) is a nonempty set $B$ together with maps $e_{i}, f_{i}: B \rightarrow B \cup\{\mathbf{0}\}$ for $i \in I$ ( $I$ indexes the simple roots, as usual, and $\mathbf{0} \notin B$ ), and wt $: B \rightarrow \Lambda$. We require $b^{\prime}=f_{i}(b)$ if and only if $b=e_{i}\left(b^{\prime}\right)$, and $\operatorname{wt}\left(f_{i}(b)\right)=\operatorname{wt}(b)-\alpha_{i}$. The maps $e_{i}$ and $f_{i}$ are called crystal operators and are represented as arrows $b \rightarrow b^{\prime}=f_{i}(b)$ colored $i$; thus they endow $B$ with the structure of a colored directed graph. For $b \in B$, we set $\varepsilon_{i}(b):=\max \left\{k \mid e_{i}^{k}(b) \neq \mathbf{0}\right\}$, and $\varphi_{i}(b):=\max \left\{k \mid f_{i}^{k}(b) \neq \mathbf{0}\right\}$. Given two $\mathfrak{g}$-crystals $B_{1}$ and $B_{2}$, we define their tensor product $B_{1} \otimes B_{2}$ as follows. As a set, $B_{1} \otimes B_{2}$ is the Cartesian product of the two sets. For $b=b_{1} \otimes b_{2} \in B_{1} \otimes B_{2}$, the weight function is simply wt $(b):=\operatorname{wt}\left(b_{1}\right)+\operatorname{wt}\left(b_{2}\right)$. The crystal operators are given by

$$
f_{i}\left(b_{1} \otimes b_{2}\right):= \begin{cases}f_{i}\left(b_{1}\right) \otimes b_{2} & \text { if } \varepsilon_{i}\left(b_{1}\right) \geq \varphi_{i}\left(b_{2}\right) \\ b_{1} \otimes f_{i}\left(b_{2}\right) & \text { otherwise, }\end{cases}
$$

and similarly for $e_{i}$. The highest weight crystal $B(\lambda)$ of highest weight $\lambda \in \Lambda^{+}$is a certain crystal with a unique element $u_{\lambda}$ such that $e_{i}\left(u_{\lambda}\right)=\mathbf{0}$ for all $i \in I$ and $\operatorname{wt}\left(u_{\lambda}\right)=\lambda$. It encodes the structure of the crystal basis of the $U_{q}(\mathfrak{g})$-irreducible representation with highest weight $\lambda$ as $q$ goes to 0 . 
A Kirillov-Reshetikhin (KR) crystal [13] is a finite crystal $B^{r, s}$ for an affine algebra, associated to a rectangle of height $r$ and width $s$, where $r \in I \backslash\{0\}$ and $s$ is any positive integer. We refer, throughout, to the untwisted affine types $A_{n-1}^{(1)}-G_{2}^{(1)}$, and only consider column shape KR crystals $B^{r, 1}$.

We now describe the models based on diagram fillings for KR crystals $B^{r, 1}$ of type $A_{n-1}^{(1)}$ and $C_{n}^{(1)}$, where $r \in\{1,2, \ldots, n-1\}$ and $r \in\{1,2, \ldots, n\}$, respectively. As a classical type $A_{n-1}$ (resp. $C_{n}$ ) crystal, the KR crystal $B^{r, 1}$ is isomorphic to the corresponding $B\left(\omega_{r}\right)$. Therefore, we can use the corresponding models in terms of fillings, as mentioned below.

In type $A_{n-1}^{(1)}$, an element $b \in B^{r, 1}$ is represented by a strictly increasing filling of a height $r$ column, with entries in $[n]:=\{1, \ldots, n\}$. We will now describe the crystal operators on a tensor product of type $A_{n-1}^{(1)} \mathrm{KR}$ crystals $B^{r, 1}$ in terms of the so-called signature rule, which is just a translation of the tensor product rule (6). To apply $f_{i}$ (or $e_{i}$ ) on $b:=b_{1} \otimes \cdots \otimes b_{k}$ in $B^{i_{1}, 1} \otimes \cdots \otimes B^{i_{k}, 1}$, consider the word with letters $i$ and $i+1$, if $1 \leq i \leq n-1$ (resp., the letters $n$ and 1 , if $i=0$ ) formed by recording these letters in $b_{1}, \ldots, b_{k}$, which are scanned from left to right and bottom to top; we make the convention that if $i=0$ and a column contains both 1 and $n$, then we discard this column. We replace the letter $i$ with the symbol + and the letter $i+1$ with - (resp., $n$ with + and 1 with - , if $i=0$ ). Then, we remove from our binary word adjacent pairs -+ , as long as this is possible. At the end of this process, we are left with a word

$$
\rho_{i}(b)=\underbrace{++\ldots+}_{x} \underbrace{--\ldots-}_{y},
$$

called the $i$-signature of $b$.

Definition 2.4 (1) If $y>0$, then $e_{i}(b)$ is obtained by replacing in $b$ the letter $i+1$ which corresponds to the leftmost - in $\rho_{i}(b)$ with the letter $i$ (resp., the letter 1 with $n$, after which we sort the column, if $i=0$ ). If $y=0$, then $e_{i}(b)=\mathbf{0}$.

(2) If $x>0$, then $f_{i}(b)$ is obtained by replacing in $b$ the letter $i$ which corresponds to the rightmost + in $\rho_{i}(b)$ with the letter $i+1$ (resp., the letter $n$ with 1 , after which we sort the column, if $i=0$ ). If $x=0$, then $f_{i}(b)=\mathbf{0}$.

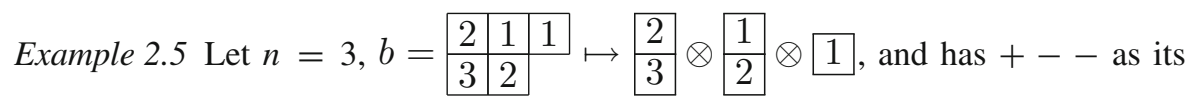
0-signature. So we have $f_{0}\left(\begin{array}{|l|l|l}\hline 2 & 1 & 1 \\
\hline 3 & 2 & \end{array}\right)=$\begin{tabular}{|l|l|l|}
\hline 1 & 1 & 1 \\
\hline 2 & 2 &
\end{tabular} .

In type $C_{n}^{(1)}$, the elements of $B^{r, 1}$ are represented by Kashiwara-Nakashima $(K N)$ columns [12] of height $r$, with entries in the set $[\bar{n}]:=\{1<\cdots<n<\bar{n}<\cdots<\overline{1}\}$, which we will now describe.

Definition 2.6 A column-strict filling $C=x_{1} \ldots x_{r}$ with entries in $[\bar{n}]$ is a KN column if there is no pair $(z, \bar{z})$ of letters in $C$ such that

$$
z=x_{p}, \bar{z}=x_{q}, q-p \leq r-z \text {. }
$$


Crystal operators $f_{i}$ and $e_{i}$ are defined on tensor products of KN columns in a similar way to type $A_{n-1}^{(1)}$. To apply $f_{i}$ (or $e_{i}$ ) on $b:=b_{1} \otimes \cdots \otimes b_{k}$ in $B^{i_{1}, 1} \otimes \cdots \otimes B^{i_{k}, 1}$, consider the word with letters $i, i+1, \bar{l}, \overline{i+1}$, if $1 \leq i \leq n-1$ (resp., the letters $n$ and $\bar{n}$, if $i=n$, or $\overline{1}$ and 1 , if $i=0$ ) formed by recording these letters in $b_{1}, \ldots, b_{k}$, which are scanned from from left to right and bottom to top; note that the letters 1 and $\overline{1}$ cannot simultaneously appear in a column, so we do not need an exception like in type $A_{n-1}^{(1)}$ if $i=0$. We replace the letters $i, \overline{i+1}$ with the symbol + and the letters $i+1, \bar{l}$ with - , if $1 \leq i \leq n-1$ (if $i=n$ we replace $n$ with + and $\bar{n}$ with - , and if $i=0$ we replace $\overline{1}$ with + and 1 with - ). We proceed like in type $A_{n-1}^{(1)}$ by canceling adjacent pairs -+ as long as possible, and we obtain the $i$-signature $\rho_{i}(b)$. The crystal operators $f_{i}$ and $e_{i}$ are again given in terms $\rho_{i}(b)$, by a similar procedure to the one in Definition 2.4. Namely, if $1 \leq i \leq n-1$, changing + to - means changing $i$ to $i+1$, if + corresponds to $i$, and changing $\overline{i+1}$ to $\bar{l}$, if + corresponds to $\overline{i+1}$; similarly changing - to + means changing $i+1$ to $i$ or $\bar{l}$ to $\overline{i+1}$. On another hand, changing + to - means changing $n$ to $\bar{n}$ if $i=n$, and changing $\overline{1}$ to 1 if $i=0$.

We will need a different definition of KN columns which was proved to be equivalent to the one above in [31].

Definition 2.7 Let $C$ be column and $I=\left\{z_{1}>\ldots>z_{k}\right\}$ the set of unbarred letters $z$ such that the pair $(z, \bar{z})$ occurs in $C$. The column $C$ can be split when there exists a set of $k$ unbarred letters $J=\left\{t_{1}>\ldots>t_{k}\right\} \subset[n]$ such that

- $t_{1}$ is the greatest letter in [n] satisfying: $t_{1}<z_{1}, t_{1} \notin C$, and $\overline{t_{1}} \notin C$,

- for $i=2, \ldots, k$, the letter $t_{i}$ is the greatest one in [n] satisfying $t_{i}<$ $\min \left(t_{i-1}, z_{i}\right), t_{i} \notin C$, and $\overline{t_{i}} \notin C$.

In this case we write

- $r C$ for the column obtained by changing $\overline{z_{i}}$ into $\overline{t_{i}}$ in $C$ for each letter $z_{i} \in I$, and by reordering if necessary,

- $l C$ for the column obtained by changing $z_{i}$ into $t_{i}$ in $C$ for each letter $z_{i} \in I$, and by reordering if necessary.

The pair $(l C, r C)$ will be called a split column, which we well sometimes denote by $l C r C$.

Example 2.8 The following is a $\mathrm{KN}$ column of height 5 in type $C_{n}$ for $n \geq 5$, together with the corresponding split column:

$$
C=\begin{array}{|l|}
\hline 4 \\
\hline 5 \\
\hline \overline{5} \\
\hline \overline{4} \\
\hline \overline{3} \\
\hline
\end{array}, \quad(l C, r C)=\begin{array}{|c|c|}
\hline 1 & 4 \\
\hline 2 & 5 \\
\hline \overline{5} & \overline{3} \\
\hline \overline{4} & \overline{2} \\
\hline \overline{3} & \overline{1} \\
\hline
\end{array}
$$

We used the fact that $\left\{z_{1}>z_{2}\right\}=\{5>4\}$, so $\left\{t_{1}>t_{2}\right\}=\{2>1\}$. 
Proposition 2.9 (1) [31] A column with entries in $[\bar{n}]$ is a KN column if and only if it can be split.

(2) [10, Theorem 5.1] The splitting is compatible with the action of the crystal operators, i.e., if $f_{i}(C)=C^{\prime}$ then $l C^{\prime} r C^{\prime}=f_{i}^{2}(l C r C)$. This holds more generally, for tensor products of columns, i.e., if $f_{i}\left(C_{1} \ldots C_{n}\right)=C_{1}^{\prime} \ldots C_{n}^{\prime}$, then $l C_{1}^{\prime} r C_{1}^{\prime} \ldots l C_{n}^{\prime} r C_{n}^{\prime}=f_{i}^{2}\left(l C_{1} r C_{1} \ldots l C_{n} r C_{n}\right)$.

In what follows we will use Definition 2.7 as the definition of $\mathrm{KN}$ columns.

We refer again to (column shape) KR crystals of arbitrary (untwisted) type. Let $\lambda=\left(\lambda_{1} \geq \lambda_{2} \geq \ldots\right)$ be a partition, which encodes a dominant weight in classical types; let $\lambda^{\prime}$ be the conjugate partition. We define

$$
B^{\otimes \lambda}:=\bigotimes_{i=1}^{\lambda_{1}} B^{\lambda_{i}^{\prime}, 1},
$$

assuming that the corresponding column shape KR crystals exist. We denote such a tensor product generically by $B$.

Remarks 2.10 (1) It is known that $B$ is connected as an affine crystal, but disconnected as a classical crystal (i.e., with the 0 -arrows removed).

(2) Let $\mathbf{p}^{\prime}$ be a composition obtained from $\mathbf{p}$ by permuting its parts. There is an affine crystal isomorphism betwee $B^{\otimes \mathbf{p}}$ and $B^{\otimes \mathbf{p}^{\prime}}$, which is unique by the previous remark. This isomorphism is called the combinatorial $R$-matrix.

We need to distinguish certain arrows in $B$, which are related to affine Demazure crystals, as we shall explain.

Definition 2.11 An arrow $b \rightarrow f_{i}(b)$ in $B$ is called a Demazure arrow if $i \neq 0$, or $i=0$ and $\varepsilon_{0}(b) \geq 1$. An arrow $b \rightarrow f_{i}(b)$ in $B$ is called a dual Demazure arrow if $i \neq 0$, or $i=0$ and $\varphi_{i}(b) \geq 2$.

Remarks 2.12 (1) By Fourier-Littelmann [3], in simply laced types, the tensor product of KR crystals $B$ is isomorphic, as a classical crystal (discard the affine 0 -arrows) with a certain Demazure crystal for the corresponding affine algebra. (Demazure modules are submodules of highest weight ones determined by a Borel subalgebra acting on an extremal weight vector.) Moreover, by [4], the 0 -arrows in the latter correspond precisely to the Demazure arrows in $B$.

(2) In the case when all of the tensor factors in $B$ are perfect crystals [9], $B$ remains connected upon removal of the non-Demazure (resp. non-dual Demazure) 0arrows.

(3) In classical types, $B^{k, 1}$ is perfect as follows: in types $A_{n-1}^{(1)}$ and $D_{n}^{(1)}$ for all $k$, in type $B_{n}^{(1)}$ only for $k \neq n$, and in type $C_{n}^{(1)}$ only for $k=n$ (using the standard indexing of the Dynkin diagram); in other words, for all the Dynkin nodes in simply laced types, and only for the nodes corresponding to the long roots in non-simply-laced types. It was conjectured in [8] that the same is true in the exceptional types. In type $G_{2}^{(1)}$ this was confirmed in [33], while for types $E_{6,7}^{(1)}$ 
and $F_{4}^{(1)}$ it was checked by computer, based on a model closely related to the quantum alcove model, see Sect. 3 and [22].

The energy function $D=D_{B}$ is a function from $B$ to the integers, defined by summing the so-called local energies of all pairs of tensor factors [8]. We will only refer here to the so-called tail energy [22], so we will not make this specification. (There are two conventions in defining the local energy of a pair of tensor factors: commuting the right one toward the head of the tensor product, or the left one toward the tail; the tail energy corresponds to the second choice.) We will only need the following property of the energy function, which defines it as an affine grading on $B$.

Theorem 2.13 [28,32] The energy is preserved by the classical crystal operators $f_{i}$, i.e., $i \neq 0$. If $b \rightarrow f_{0}(b)$ is a dual Demazure arrow, then $D\left(f_{0}(b)\right)=D(b)-1$.

Remarks 2.14 Theorem 2.13 shows that the energy is determined up to a constant on the connected components of the subgraph of the affine crystal $B$ containing only the dual Demazure arrows. See also Remark 2.12 (2).

One can define a statistic called charge on the model based on KN columns for $B^{\otimes \lambda}$ in types $A$ and $C$. This was done in [18], by translating a certain statistic in the Ram-Yip formula for Macdonald polynomials (i.e., the height statistic in (11)) to the model based on KN columns, via certain bijections recalled in Sect. 4, cf. Remarks 4.8 (2) and 4.23 (2). In type $A$, this procedure leads to the same statistic that was originally defined by Lascoux and Schützenberger [14]. A similar procedure to the one in [18] is under investigation in type $B$ in [1]. The charge statistic is related to the energy function by the following theorem.

Theorem 2.15 [25,29] Let $B^{\otimes \lambda}$ be a tensor product of KR crystals in type $A_{n-1}^{(1)}$ or type $C_{n}^{(1)}$. For all $b \in B^{\otimes \lambda}$, we have $D(b)=-\operatorname{charge}(b)$.

The charge gives a much easier method to compute the energy than the recursive one based on Theorem 2.13. See also Theorem 3.24 (2) for a much more general result.

\section{The quantum alcove model}

In this section we construct the quantum alcove model and study its main properties.

\section{1 $\lambda$-Chains and admissible subsets}

We say that two alcoves are adjacent if they are distinct and have a common wall. Given a pair of adjacent alcoves $A$ and $B$, we write $A \stackrel{\beta}{\longrightarrow} B$ if the common wall is of the form $H_{\beta, k}$ and the root $\beta \in \Phi$ points in the direction from $A$ to $B$.

Definition 3.1 [23] An alcove path is a sequence of alcoves $\left(A_{0}, A_{1}, \ldots, A_{m}\right)$ such that $A_{j-1}$ and $A_{j}$ are adjacent, for $j=1, \ldots m$. We say that an alcove path is reduced if it has minimal length among all alcove paths from $A_{0}$ to $A_{m}$. 


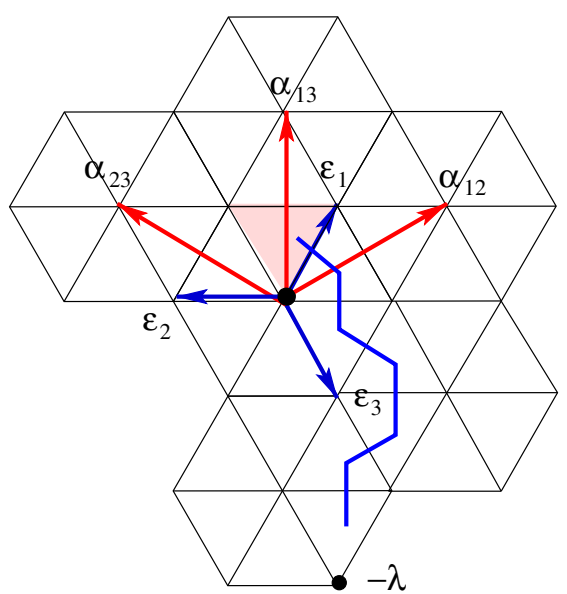

(a)

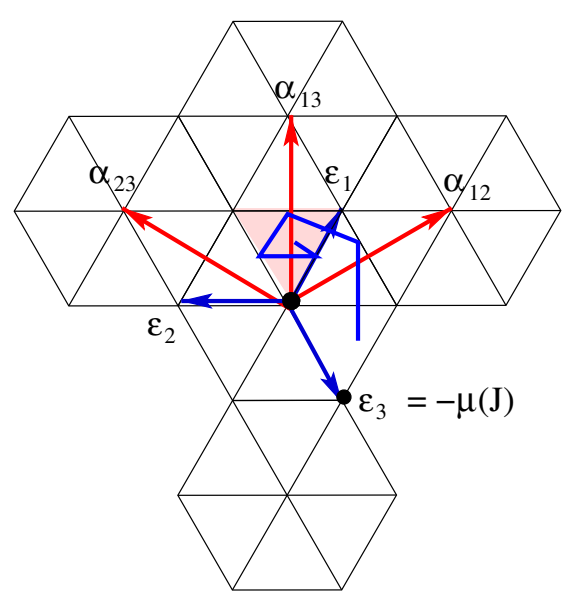

(b)

Fig. 1 Unfolded and folded $\lambda$-chain. a $\Gamma$ for $\lambda=3 \varepsilon_{1}+2 \varepsilon_{2}$. b $\Gamma(J)$ for $J=\{1,2,3,5\}$

Let $A_{\lambda}=A_{\circ}+\lambda$ be the translation of the fundamental alcove $A_{\circ}$ by the weight $\lambda$.

Definition 3.2 [23] The sequence of roots $\left(\beta_{1}, \beta_{2}, \ldots, \beta_{m}\right)$ is called a $\lambda$-chain if

$$
A_{0}=A_{\circ} \stackrel{-\beta_{1}}{\longrightarrow} A_{1} \stackrel{-\beta_{2}}{\longrightarrow} \cdots \stackrel{-\beta_{m}}{\longrightarrow} A_{m}=A_{-\lambda}
$$

is a reduced alcove path.

We now fix a dominant weight $\lambda$ and an alcove path $\Pi=\left(A_{0}, \ldots, A_{m}\right)$ from $A_{0}=A_{\circ}$ to $A_{m}=A_{-\lambda}$. Note that $\Pi$ is determined by the corresponding $\lambda$-chain $\Gamma:=\left(\beta_{1}, \ldots, \beta_{m}\right)$, which consists of positive roots. A specific choice of a $\lambda$-chain, called a lex $\lambda$-chain and denoted $\Gamma_{\text {lex }}$, is given in [24] [Proposition 4.2]; this choice depends on a total order on the simple roots. We let $r_{i}:=s_{\beta_{i}}$, and let $\widehat{r_{i}}$ be the affine reflection in the hyperplane containing the common face of $A_{i-1}$ and $A_{i}$, for $i=1, \ldots, m$; in other words, $\widehat{r}_{i}:=s_{\beta_{i},-l_{i}}$, where $l_{i}:=\left|\left\{j<i ; \beta_{j}=\beta_{i}\right\}\right|$. We define $\widetilde{l}_{i}:=\left\langle\lambda, \beta_{i}^{\vee}\right\rangle-l_{i}=\left|\left\{j \geq i ; \beta_{j}=\beta_{i}\right\}\right|$.

Example 3.3 Consider the dominant weight $\lambda=3 \varepsilon_{1}+2 \varepsilon_{2}$ in the root system $A_{2}$ (cf. Sect. 4.1 and the notation therein). A $\lambda$-chain is $\Gamma=\left(\alpha_{23}, \alpha_{13}, \alpha_{23}, \alpha_{13}, \alpha_{12}, \alpha_{13}\right)$. The corresponding $l_{i}$ are $(0,0,1,1,0,2)$ and $\widetilde{l}_{i}$ are $\{2,3,1,2,1,1\}$. The alcove path is shown in Fig. 1a; here $A_{\circ}$ is shaded, and $A_{-\lambda}$ is the alcove at the end of the path.

Let $J=\left\{j_{1}<j_{2}<\cdots<j_{s}\right\}$ be a subset of $[m]$. The elements of $J$ are called folding positions. We fold $\Pi$ in the hyperplanes corresponding to these positions and obtain a folded path, see Example 3.6 and Fig. 1b. Like $\Pi$, the folded path can be recorded by a sequence of roots, namely $\Gamma(J)=\left(\gamma_{1}, \gamma_{2}, \ldots, \gamma_{m}\right)$, where

$$
\gamma_{k}:=r_{j_{1}} r_{j_{2}} \ldots r_{j_{p}}\left(\beta_{k}\right)
$$


with $j_{p}$ the largest folding position less than $k$. We define $\gamma_{\infty}:=r_{j_{1}} r_{j_{2}} \ldots r_{j_{s}}(\rho)$. Upon folding, the hyperplane separating the alcoves $A_{k-1}$ and $A_{k}$ in $\Pi$ is mapped to

$$
H_{\left|\gamma_{k}\right|,-l_{k}^{J}}=\widehat{r}_{j_{1}} \widehat{r}_{j_{2}} \ldots \widehat{r}_{j_{p}}\left(H_{\beta_{k},-l_{k}}\right)
$$

for some $l_{k}^{J}$, which is defined by this relation.

Given $i \in J$, we say that $i$ is a positive folding position if $\gamma_{i}>0$, and a negative folding position if $\gamma_{i}<0$. We denote the positive folding positions by $J^{+}$, and the negative ones by $J^{-}$. We call $\mu=\mu(J):=-\widehat{r}_{j_{1}} \widehat{r}_{j_{2}} \ldots \widehat{r}_{j_{s}}(-\lambda)$ the weight of $J$. We define

$$
\operatorname{height}(J):=\sum_{j \in J^{-}} \widetilde{l}_{j}
$$

Definition 3.4 A subset $J=\left\{j_{1}<j_{2}<\cdots<j_{s}\right\} \subseteq[m]$ (possibly empty) is an admissible subset if we have the following path in the quantum Bruhat graph on $W$ :

$$
1 \stackrel{\beta_{j_{1}}}{\longrightarrow} r_{j_{1}} \stackrel{\beta_{j_{2}}}{\longrightarrow} r_{j_{1}} r_{j_{2}} \stackrel{\beta_{j_{3}}}{\longrightarrow} \cdots \stackrel{\beta_{j_{s}}}{\longrightarrow} r_{j_{1}} r_{j_{2}} \cdots r_{j_{s}}
$$

We call $\Gamma(J)$ an admissible folding. We let $\mathcal{A}(\Gamma)$ be the collection of admissible subsets.

Remarks 3.5 If we restrict to admissible subsets for which the path (12) has no down steps, we recover the classical alcove model in [23,24].

Example 3.6 We continue Example 3.3. Let $J=\{1,2,3,5\}$, then $\Gamma(J)=$ $\left(\alpha_{23}, \alpha_{12}, \alpha_{31}, \alpha_{23}, \alpha_{21}, \alpha_{13}\right)$. The folded path is shown in Fig. 1b. We have $J^{+}=$ $\{1,2\}, J^{-}=\{3,5\}, \mu(J)=-\varepsilon_{3}$, and height $(J)=2$. In Sect. 4.1 we will describe an easy way to verify that $J$ is admissible.

\subsection{Crystal operators}

In this section we define the crystal operators on $\mathcal{A}(\Gamma)$. Given $J \subseteq[m]$ and $\alpha \in \Phi$, we will use the following notation:

$$
I_{\alpha}=I_{\alpha}(J):=\left\{i \in[m] \mid \gamma_{i}= \pm \alpha\right\}, \quad \widehat{I}_{\alpha}=\widehat{I}_{\alpha}(J):=I_{\alpha} \cup\{\infty\}
$$

and $l_{\alpha}^{\infty}:=\left\langle\mu(J), \operatorname{sgn}(\alpha) \alpha^{\vee}\right\rangle$. The following graphical representation of the heights $l_{i}^{J}$ for $i \in I_{\alpha}$ and $l_{\alpha}^{\infty}$ is useful for defining the crystal operators. Let

$$
\widehat{I}_{\alpha}=\left\{i_{1}<i_{2}<\cdots<i_{n}<i_{n+1}=\infty\right\} \text { and } \varepsilon_{i}:=\left\{\begin{array}{ll}
1 & \text { if } i \notin J \\
-1 & \text { if } i \in J
\end{array}\right. \text {. }
$$



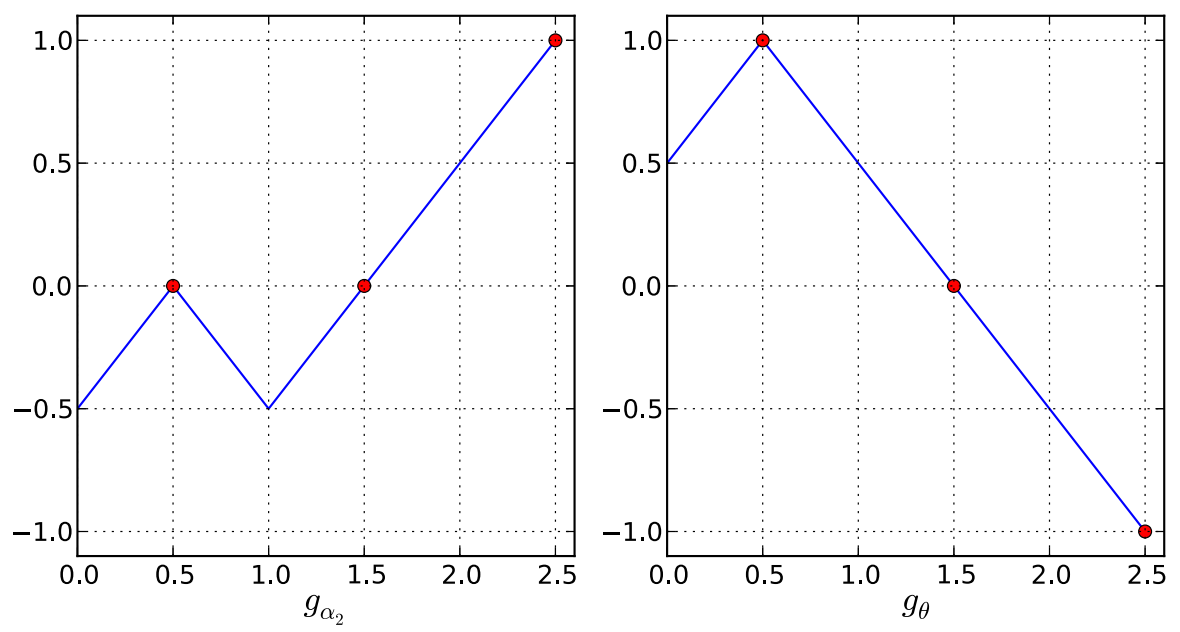

Fig. 2 Examples of $g_{\alpha}$

If $\alpha>0$, we define the continuous piecewise linear function $g_{\alpha}:\left[0, n+\frac{1}{2}\right] \rightarrow \mathbb{R}$ by

$$
g_{\alpha}(0)=-\frac{1}{2}, \quad g_{\alpha}^{\prime}(x)= \begin{cases}\operatorname{sgn}\left(\gamma_{i_{k}}\right) & \text { if } x \in\left(k-1, k-\frac{1}{2}\right), k=1, \ldots, n \\ \varepsilon_{i_{k}} \operatorname{sgn}\left(\gamma_{i_{k}}\right) & \text { if } x \in\left(k-\frac{1}{2}, k\right), k=1, \ldots, n \\ \operatorname{sgn}\left(\left\langle\gamma_{\infty}, \alpha^{\vee}\right\rangle\right) & \text { if } x \in\left(n, n+\frac{1}{2}\right) .\end{cases}
$$

If $\alpha<0$, we define $g_{\alpha}$ to be the graph obtained by reflecting $g_{-\alpha}$ in the $x$-axis. By [24][Propositions 5.3 and 5.5], for any $\alpha$ we have

$$
\operatorname{sgn}(\alpha) l_{i_{k}}^{J}=g_{\alpha}\left(k-\frac{1}{2}\right), k=1, \ldots, n, \text { and } \operatorname{sgn}(\alpha) l_{\alpha}^{\infty}:=\left\langle\mu(J), \alpha^{\vee}\right\rangle=g_{\alpha}\left(n+\frac{1}{2}\right) \text {. }
$$

Example 3.7 We continue Example 3.6. The graphs of $g_{\alpha_{2}}$ and $g_{\theta}$ are given in Fig. 2.

Let $J$ be an admissible subset. Let $\delta_{i, j}$ be the Kronecker delta function. Fix $p$ in $\{0, \ldots, r\}$, so $\alpha_{p}$ is a simple root if $p>0$, or $\theta$ if $p=0$. Let $M$ be the maximum of $g_{\alpha_{p}}$. Let $m$ be the minimum index $i$ in $\widehat{I}_{\alpha_{p}}$ for which we have $\operatorname{sgn}\left(\alpha_{p}\right) l_{i}^{J}=M$. By Proposition 3.22, if $M \geq \delta_{p, 0}$, then we have either $m \in J$ or $m=\infty$; furthermore, if $M>\delta_{p, 0}$, then $m$ has a predecessor $k$ in $\widehat{I}_{\alpha_{p}}$, and we have $k \notin J$. We define

$$
f_{p}(J):= \begin{cases}(J \backslash\{m\}) \cup\{k\} & \text { if } M>\delta_{p, 0} \\ \mathbf{0} & \text { otherwise }\end{cases}
$$

Now we define $e_{p}$. Again let $M:=\max g_{\alpha_{p}}$. Assuming that $M>\left\langle\mu(J), \alpha_{p}^{\vee}\right\rangle$, let $k$ be the maximum index $i$ in $I_{\alpha_{p}}$ for which we have $\operatorname{sgn}\left(\alpha_{p}\right) l_{i}^{J}=M$, and let $m$ be the successor of $k$ in $\widehat{I}_{\alpha_{p}}$. Assuming also that $M \geq \delta_{p, 0}$, by Proposition 3.23 we have 
$k \in J$, and either $m \notin J$ or $m=\infty$. Define

$$
e_{p}(J):= \begin{cases}(J \backslash\{k\}) \cup\{m\} & \text { if } M>\left\langle\mu(J), \alpha_{p}^{\vee}\right\rangle \text { and } M \geq \delta_{p, 0} \\ \mathbf{0} & \text { otherwise. }\end{cases}
$$

In the above definitions, we use the convention that $J \backslash\{\infty\}=J \cup\{\infty\}=J$.

Example 3.8 We continue Example 3.7. We find $f_{2}(J)$ by noting that $\widehat{I}_{\alpha_{2}}=\{1,4, \infty\}$. From $g_{\alpha_{2}}$ in Fig. 2 we can see that the heights $l_{i}^{J}$ and $l_{\alpha_{2}}^{\infty}$ corresponding to these positions are $0,0,1$, so $k=4, m=\infty$, and $f_{2}(J)=J \cup\{4\}=\{1,2,3,4,5\}$. We can see from Fig. 2 that the maximum of $g_{\theta}=1$, hence $f_{0}(J)=\mathbf{0}$. To compute $e_{0}(J)$ observe that $\widehat{I_{\theta}}=\{3,6\}$ with $k=3$ and $m=6$. So $e_{0}(J)=(J \backslash\{k\}) \cup\{m\}=\{1,2,5,6\}$.

The following theorem is one of our main results, and will be proved in Sect. 3.3.

Theorem 3.9 (1) If $J$ is an admissible subset and if $f_{p}(J) \neq \mathbf{0}$, then $f_{p}(J)$ is also an admissible subset. Similarly for $e_{p}(J)$. Moreover, $f_{p}(J)=J^{\prime}$ if and only if $e_{p}\left(J^{\prime}\right)=J$.

(2) We have $\mu\left(f_{p}(J)\right)=\mu(J)-\alpha_{p}$. Moreover, if $M \geq \delta_{p, 0}$, then

$$
\varphi_{p}(J)=M-\delta_{p, 0}, \quad \varepsilon_{p}(J)=M-\left\langle\mu(J), \alpha_{p}^{\vee}\right\rangle
$$

while otherwise $\varphi_{p}(J)=\varepsilon_{p}(J)=0$.

\subsection{Proofs}

In this section we collect necessary results for the proof of Theorem 3.9. The techniques are similar to those in [24]; we go in detail over the parts of the proofs where there are notable differences, and we refer to the mentioned paper for the remaining parts.

Lemma 3.10 Let $w \in W, \alpha$ a simple root or $\theta$, and $\beta$ a positive root. If we have $w \longrightarrow w s_{\beta}$, as well as $w^{-1}(\alpha)>0$ and $s_{\beta} w^{-1}(\alpha)<0$, then $w^{-1}(\alpha)=\beta$.

Proof If $s_{\alpha} w=w s_{\beta}$, then $w^{-1}(\alpha)= \pm \beta$, and $w^{-1}(\alpha)>0$ implies $w^{-1}(\alpha)=\beta$. Suppose by way of contradiction that $s_{\alpha} w \neq w s_{\beta}$. First suppose $\alpha$ is a simple root. Since $w^{-1}(\alpha)>0$, then $w \lessdot s_{\alpha} w$. By assumption we have $w \longrightarrow w s_{\beta}$, hence by Lemma 2.2 we have $w s_{\beta} \lessdot s_{\alpha} w s_{\beta}$. But $s_{\beta} w^{-1}(\alpha)<0$ implies $s_{\alpha} w s_{\beta} \lessdot w s_{\beta}$, which is a contradiction.

Suppose $\alpha=\theta$. Since $w^{-1}(\theta)>0$, we deduce $w \triangleleft s_{\theta} w$, by Lemma 2.1, and then $w s_{\beta} \triangleleft s_{\theta} w s_{\beta}$, by Lemma 2.3. On another hand, since $s_{\beta} w^{-1}(\theta)<0$, Lemma 2.1 implies that $s_{\theta} w s_{\beta} \triangleleft w s_{\beta}$, which is a contradiction.

Lemma 3.11 Let $J=\left\{j_{1}<j_{2}<\ldots<j_{s}\right\}$ be an admissible subset. Assume that $r_{j_{a}} \ldots r_{j_{1}}(\alpha)>0$ and $r_{j_{b}} \ldots r_{j_{1}}(\alpha)<0$, where $\alpha$ is a simple root or $\theta$, and $0 \leq a<b$ (if $a=0$, then the first condition is void). Then there exists $i$ with $a \leq i<b$ such that $\gamma_{j_{i+1}}=\alpha$. 
Proof Find $i$ with $a \leq i<b$ such that $r_{j_{i}} \ldots r_{j_{1}}(\alpha)>0$ and $r_{j_{i+1}} \ldots r_{j_{1}}(\alpha)<$ 0 . By Lemma 3.10, we have $\beta_{j_{i+1}}=r_{j_{i}} \ldots r_{j_{1}}(\alpha)$. This means that $\gamma_{j_{i+1}}=$ $r_{j_{1}} \ldots r_{j_{i}}\left(\beta_{j_{i+1}}\right)=\alpha$.

Proposition 3.12 Let $J=\left\{j_{1}<j_{2}<\cdots<j_{s}\right\}$ be an admissible subset. Assume that $\alpha$ is a simple root or $\theta$, with $I_{\alpha} \neq \emptyset$. Let $m \in I_{\alpha}$ be an element for which its predecessor $k$ (in $I_{\alpha}$ ) satisfies $\left(\gamma_{k}, \varepsilon_{k}\right) \in\{(\alpha, 1),(-\alpha,-1)\}$. Then we have $\gamma_{m}=\alpha$.

Proof First suppose that $\left(\gamma_{k}, \varepsilon_{k}\right)=(\alpha, 1)$. Assume that $\gamma_{m}=-\alpha$. Let us define the index $b$ by the condition $j_{b}<m \leq j_{b+1}$ (possibly $b=s$, in which case the second inequality is dropped). We define the index $a$ by the condition $j_{a}<k<j_{a+1}$ (possibly $a=0$, in which case the first inequality is dropped). We clearly have $r_{j_{1}} \ldots r_{j_{b}}\left(\beta_{m}\right)=-\alpha$, which implies $r_{j_{b}} \ldots r_{j_{1}}(\alpha)<0$. We also have $r_{j_{1}} \ldots r_{j_{a}}\left(\beta_{k}\right)=$ $\alpha$, so $r_{j_{a}} \ldots r_{j_{1}}(\alpha)>0$ (hence $a<b$ ). Note that if $\alpha=\theta$, then $a>0$. We can now apply Lemma 3.11 to conclude that $\gamma_{j_{i}}=\alpha$ for some $i \in[a+1, b]$. Since $k<j_{a+1} \leq j_{b}<m$, we contradicted the assumption that $\gamma_{k}$ is the predecessor of $\gamma_{m}$ in $I_{\alpha}$.

Now suppose that $\left(\gamma_{k}, \varepsilon_{k}\right)=(-\alpha,-1)$. Assume that $\gamma_{m}=-\alpha$ and define $b$ as in the previous case. Again we have $r_{j_{b}} \ldots r_{j_{1}}(\alpha)<0$. Define $a$ by the condition $j_{a}=k<j_{a+1}$. Hence $r_{j_{1}} \ldots r_{j_{a-1}}\left(\beta_{j_{a}}\right)=-\alpha$, so $r_{j_{a}} \ldots r_{j_{1}}(\alpha)>0$. This leads to a contradiction by a similar reasoning to the one above.

Proposition 3.13 Let $J$ be an admissible subset. Assume that $\alpha$ is a simple root for which $I_{\alpha} \neq \emptyset$. Let $m \in I_{\alpha}$ be the minimum of $I_{\alpha}$. Then we have $\gamma_{m}=\alpha$.

Proof The proof of Proposition 3.12 carries through with $a=0$.

Proposition 3.14 Let $J=\left\{j_{1}<j_{2}<\cdots<j_{s}\right\}$ be an admissible subset. Assume that $\alpha$ is a simple root or $\theta$. Suppose that $I_{\alpha} \neq \emptyset$, and $\left(\gamma_{m}, \varepsilon_{m}\right) \in\{(\alpha, 1),(-\alpha,-1)\}$ for $m=\max I_{\alpha}$. Then we have $\left\langle\gamma_{\infty}, \alpha^{\vee}\right\rangle>0$.

Proof Assume that the conclusion fails, which means that $r_{j_{s}} \ldots r_{j_{1}}(\alpha)<0$. First suppose that $\left(\gamma_{m}, \varepsilon_{m}\right)=(\alpha, 1)$. Define the index $a$ by the condition $j_{a}<m<j_{a+1}$. (If $a=0$ or $a=s$ one of the two inequalities is dropped). We have $r_{j_{1}} \ldots r_{j_{a}}\left(\beta_{m}\right)=\alpha$, so $r_{j_{a}} \ldots r_{j_{1}}(\alpha)>0$ (hence $a \neq s$ ). Note that if $\alpha=\theta$, then $a>0$. We now apply Lemma 3.11 to conclude that $\gamma_{j_{i}}=\alpha$ for $i \in[a+1, s]$. Since $m<j_{a+1} \leq j_{s}$, this contradicts that $m=\max I_{\alpha}$.

Now suppose that $\left(\gamma_{m}, \varepsilon_{m}\right)=(-\alpha,-1)$. In this case we define the index $a$ by $j_{a}=m<j_{a+1}$. We have $r_{j_{1}} \ldots r_{j_{a-1}}\left(\beta_{j_{a}}\right)=-\alpha$, so $r_{j_{a}} \ldots r_{j_{1}}(\alpha)>0$. This leads to a contradiction by a similar reasoning to the one above.

Proposition 3.15 Let $J$ be an admissible subset. Assume that, for some simple root $\alpha$, we have $I_{\alpha}=\emptyset$. Then $\left\langle\gamma_{\infty}, \alpha^{\vee}\right\rangle>0$.

Proof The proof of Proposition 3.14 carries through with $a=0$.

Let us now fix a simple root $\alpha$. We will rephrase some of the above results in a simple way in terms of $g_{\alpha}$, and we will deduce some consequences. Assume that $I_{\alpha}=$ 
$\left\{i_{1}<i_{2}<\cdots<i_{n}\right\}$, so that $g_{\alpha}$ is defined on $\left[0, n+\frac{1}{2}\right]$, and let $M$ be the maximum of $g_{\alpha}$. Note first that the function $g_{\alpha}$ is determined by the sequence $\left(\sigma_{1}, \ldots, \sigma_{n+1}\right)$, where $\sigma_{j}=\left(\sigma_{j, 1}, \sigma_{j, 2}\right):=\left(\operatorname{sgn}\left(\gamma_{i_{j}}\right), \varepsilon_{i_{j}} \operatorname{sgn}\left(\gamma_{i_{j}}\right)\right)$ for $1 \leq j \leq n$, and $\sigma_{n+1}=\sigma_{n+1,1}:=$ $\operatorname{sgn}\left(\left\langle\gamma_{\infty}, \alpha^{\vee}\right\rangle\right)$. From Propositions 3.12, 3.13, 3.14 and 3.15 we have the following restrictions.

(C1) $\sigma_{1,1}=1$.

(C2) $\sigma_{j, 2}=1 \Rightarrow \sigma_{j+1,1}=1$.

Proposition 3.16 If $g_{\alpha}(x)=M$, then $M \in \mathbb{Z}_{\geq 0}, x=m+\frac{1}{2}$ for $0 \leq m \leq n$, and $\sigma_{m+1} \in\{(1,-1), 1\}$.

Proof By (C1), we have $M \geq 0$, therefore $g_{\alpha}(0)=-\frac{1}{2} \neq M$. For $m \in\{1, \ldots, n\}$, if $g_{\alpha}(m)=M$ then $\sigma_{m, 2}=1$, and (C2) leads to a contradiction. The last statement is obvious.

We use Proposition 3.16 implicitly in the proof of Proposition 3.17 and Proposition 3.18 .

Proposition 3.17 Assume that $M>0$, and let $m$ be such that $m+\frac{1}{2}=\min g_{\alpha}^{-1}(M)$. We have $m>0, \sigma_{m}=(1,1)$, and $g_{\alpha}\left(m-\frac{1}{2}\right)=M-1$. Moreover, we have $g_{\alpha}(x) \leq$ $M-1$ for $0 \leq x \leq m-\frac{1}{2}$.

Proof By (C1) we have $g_{\alpha}\left(\frac{1}{2}\right)=0$, so $m>0$. If $\sigma_{m} \in\{(-1,-1),(1,-1)\}$, then we have $g_{\alpha}\left(m-\frac{1}{2}\right)=M$, which contradicts the definition of $m$. If $\sigma_{m}=(-1,1)$, then $g_{\alpha}(m-1)=M-\frac{1}{2}>-\frac{1}{2}$. By (C1) we have $m \geq 2$, and by (C2) we have $\sigma_{m-1,2}=-1$. This implies that $g_{\alpha}\left(m-\frac{3}{2}\right)=M$, contradicting the definition of $m$. Hence $\sigma_{m}=(1,1)$.

Suppose by way of contradiction that the last statement in the corollary fails. Then there exists a $k$ with $1 \leq k \leq m-1$ such that $g_{\alpha}(k-1)=M-\frac{1}{2}>-\frac{1}{2}$ and $\sigma_{k, 1}=-1$. Condition (C1) implies that $k \geq 2$ and Condition (C2) implies $\sigma_{k-1,2}=-1$. This implies $g_{\alpha}\left(k-\frac{3}{2}\right)=M$, contradicting the definition of $m$.

Proposition 3.18 Assume that $M>g_{\alpha}\left(n+\frac{1}{2}\right)$, and let $k$ be such that $k-\frac{1}{2}=$ $\max g_{\alpha}^{-1}(M)$. We have $k \leq n, \sigma_{k+1} \in\{(-1,-1),-1\}$, and $g_{\alpha}\left(k+\frac{1}{2}\right)=M-1$. Moreover, we have $g_{\alpha}(x) \leq M-1$ for $k+\frac{1}{2} \leq x \leq n+\frac{1}{2}$.

Proof Since $M>g_{\alpha}\left(n+\frac{1}{2}\right)$, it follows that $k \leq n$. If $\sigma_{k+1} \in\{(1,1),(1,-1), 1\}$ then $g_{\alpha}\left(k+\frac{1}{2}\right)=M$, contradicting the choice of $k$. If $\sigma_{k+1}=(-1,1)$, then by (C2) we have $\sigma_{k+2,1}=1$, and $g_{\alpha}\left(k+\frac{3}{2}\right)=M$, contradicting the choice of $k$. Hence $\sigma_{k+1} \in\{(-1,-1),-1\}$.

Suppose by way of contradiction the last statement in the corollary fails. Then there exists an $m$ with $k+2 \leq m \leq n$ such that $g_{\alpha}(m)=M-\frac{1}{2}$ and $\sigma_{m, 2}=1$. Condition (C2) implies that $\sigma_{m+1,1}=1$, so $g_{\alpha}\left(m+\frac{1}{2}\right)=M$, contradicting the choice of $k$.

We now consider $g_{\theta}$. Since $\theta<0$, the definition of the piecewise linear function $g_{\theta}$ requires us to define its linear steps by $\sigma_{j}=\left(\sigma_{j, 1}, \sigma_{j, 2}\right):=\left(-\operatorname{sgn}\left(\gamma_{i_{j}}\right),-\varepsilon_{i_{j}} \operatorname{sgn}\left(\gamma_{i_{j}}\right)\right)$ 
for $1 \leq j \leq n$, and $\sigma_{n+1}=\sigma_{n+1,1}:=\operatorname{sgn}\left(\left\langle\gamma_{\infty}, \theta^{\vee}\right\rangle\right)$. From Propositions 3.12 and 3.14 we conclude that condition (C2) holds for $g_{\theta}$. We can replace condition (C1) by restricting to admissible subsets $J$ where $M$ is large enough, as we will now explain. In the proof of Proposition 3.16, condition $(\mathrm{C} 1)$ is needed to conclude that $g_{\alpha}(0) \neq M$. It is possible that $g_{\theta}(0)=M$, but if we restrict to $g_{\theta}$ where $M \geq 1$ we can conclude that $g_{\theta}(0)=\frac{1}{2} \neq M$, and the rest of the proof follows through. In the proof of Proposition 3.17, condition (C1) first implies $m \geq 1$; we can conclude the same thing if we assume $M \geq 2$, since $g_{\theta}\left(\frac{1}{2}\right) \leq 1$. Then we need to derive $m \geq 2$ from $g_{\theta}(m-1)=M-\frac{1}{2}$; again, if $M \geq 2$, then $M-\frac{1}{2}>g_{\theta}(0)=\frac{1}{2}$, so $m-1>0$. Note that Proposition 3.18 depends on Proposition 3.16 so we need to assume $M \geq 1$ here too. We have therefore proved the following propositions.

Proposition 3.19 Suppose $M \geq 1$. If $g_{\theta}(x)=M$, then $M \in \mathbb{Z}_{\geq 1}, x=m+\frac{1}{2}$ for $0 \leq m \leq n$, and $\sigma_{m+1} \in\{(1,-1), 1\}$.

Proposition 3.20 Assume that $M \geq 2$, and let $m$ be such that $m+\frac{1}{2}=\min g_{\theta}^{-1}(M)$. We have $m>0, \sigma_{m}=(1,1)$, and $g_{\theta}\left(m-\frac{1}{2}\right)=M-1$. Moreover, we have $g_{\theta}(x) \leq$ $M-1$ for $0 \leq x \leq m-\frac{1}{2}$.

Proposition 3.21 Assume $M \geq 1$, and also that $M>g_{\theta}\left(n+\frac{1}{2}\right)$. Let $k$ be such that $k-\frac{1}{2}=\max g_{\theta}^{-1}(M)$. We have $k \leq n, \sigma_{k+1} \in\{(-1,-1),-1\}$, and $g_{\theta}\left(k+\frac{1}{2}\right)=$ $M-1$. Moreover, we have $g_{\theta}(x) \leq M-1$ for $k+\frac{1}{2} \leq x \leq n+\frac{1}{2}$.

Recall from from Sect. 3.2 the definitions of the finite sequences $I_{\alpha}(J)$ and $\widehat{I_{\alpha}}(J)$, where $\alpha$ is a root, of $g_{\alpha}$, as well as the related notation.

Fix $p$, so $\alpha_{p}$ is a simple root if $p>0$, or $\theta$ if $p=0$. Let $M$ be the maximum of $g_{\alpha_{p}}$, and suppose that $M \geq \delta_{p, 0}$. Note this is always true for $p \neq 0$ by Proposition 3.16. Let $m$ be the minimum index $i$ in $\widehat{I}_{\alpha_{p}}(J)$ for which we have $\operatorname{sgn}\left(\alpha_{p}\right) l_{i}^{J}=M$. The following proposition is an immediate consequence of Propositions 3.16, 3.17, 3.19, 3.20.

Proposition 3.22 Given the above setup, the following hold.

(1) If $m \neq \infty$, then $\gamma_{m}=\alpha_{p}$ and $m \in J$.

(2) If $M>\delta_{p, 0}$, then $m$ has a predecessor $k$ in $\widehat{I}_{\alpha_{p}}(J)$ such that

$$
\gamma_{k}=\alpha_{p}, k \notin J, \text { and } \operatorname{sgn}\left(\alpha_{p}\right) l_{k}^{J}=M-1
$$

Now assume that $M>\left\langle\mu(J), \alpha_{p}^{\vee}\right\rangle$. Let $k$ be the maximum index $i$ in $I_{\alpha_{p}}(J)$ for which we have $\operatorname{sgn}\left(\alpha_{p}\right) l_{i}^{J}=M$, and let $m$ be the successor of $k$ in $\widehat{I}_{\alpha_{p}}(J)$. The following analog of Proposition 3.22 is proved in a similar way, based on Propositions 3.16, 3.18, 3.19, 3.21.

Proposition 3.23 Given the above setup, and assuming also that $M \geq \delta_{p, 0}$, the following hold. 
(1) We have $\gamma_{k}=\alpha_{p}$ and $k \in J$.

(2) If $m \neq \infty$, then

$$
\gamma_{m}=-\alpha_{p}, m \notin J, \text { and } \operatorname{sgn}\left(\alpha_{p}\right) l_{m}^{J}=M-1 .
$$

Proof of Theorem 3.9 Suppose $p \neq 0$. We consider $f_{p}$ first. The cases corresponding to $m \neq \infty$ and $m=\infty$ can be proved in similar ways, so we only consider the first case. Let $J=\left\{j_{1}<j_{2}<\ldots<j_{s}\right\}$, and let $w_{i}:=r_{j_{1}} r_{j_{2}} \ldots r_{j_{i}}$. Based on Proposition 3.22, let $a<b$ be such that

$$
j_{a}<k<j_{a+1}<\cdots<j_{b}=m<j_{b+1}
$$

if $a=0$ or $b+1>s$, then the corresponding indices $j_{a}$, respectively $j_{b+1}$, are missing. To show that $(J \backslash\{m\}) \cup\{k\}$ is an admissible subset, it is enough to prove that we have the path in the quantum Bruhat graph

$$
w_{a} \longrightarrow w_{a} r_{k} \longrightarrow w_{a} r_{k} r_{j a+1} \longrightarrow \cdots \longrightarrow w_{a} r_{k} r_{j_{a+1}} \ldots r_{j_{b-1}}=w_{b}
$$

By our choice of $k$, we have

$$
w_{a}\left(\beta_{k}\right)=\alpha_{p} \Longleftrightarrow w_{a}^{-1}\left(\alpha_{p}\right)=\beta_{k}>0 \Longleftrightarrow w_{a} \lessdot s_{p} w_{a}=w_{a} r_{k} .
$$

So we can rewrite (17) as

$$
w_{a} \longrightarrow s_{p} w_{a} \longrightarrow s_{p} w_{a+1} \longrightarrow \cdots \longrightarrow s_{p} w_{b-1}=w_{b}
$$

We will now prove that (19) is a path in the quantum Bruhat graph. Observe that, for $a<i \leq b$, we have

$$
s_{p} w_{i-1}=w_{i}=w_{i-1} r_{j_{i}} \Longleftrightarrow w_{i-1}\left(\beta_{j_{i}}\right)= \pm \alpha_{p} \Longleftrightarrow j_{i} \in I_{\alpha} .
$$

Our choice of $k$ and $b$ implies that we have

$$
s_{p} w_{i-1} \neq w_{i} \text { for } a<i<b, \quad \text { and } s_{p} w_{b-1}=w_{b} .
$$

Since $J$ is admissible, we have

$$
w_{i-1} \longrightarrow w_{i}
$$

Starting from (18), and then using (20)-(21), we can apply Lemma 2.2 repeatedly to conclude that

$$
s_{p} w_{i-1} \longrightarrow s_{p} w_{i} \text { and } w_{i} \lessdot s_{p} w_{i}, \quad \text { for } a<i<b \text {. }
$$

The proof for $e_{p}(J)$ is similar. The main difference is that we need the "if" part of Lemma 2.2, whereas above we used the "only if" part.

The above proof follows through for $p=0$, based on Lemma 2.1, which is used to derive the analog of (18), and Lemma 2.3, which replaces Lemma 2.2. 
We can prove that $f_{p}(J)=J^{\prime}$ if and only if $e_{p}\left(J^{\prime}\right)=J$ based on [24] [Proposition 7.4 (1)]; this still holds in the above setup (for any $p$, including $p=0$ ), based on Propositions 3.16-3.21. The same result can be invoked to derive the formulas for $\varphi_{p}(J)$ and $\varepsilon_{p}(J)$.

In order to show that $f_{p}$ changes weights by $-\alpha_{p}$, the proof of [24][Proposition 7.1 (3)] can be applied in our context. In essence, we note that $\mu\left(f_{p}(J)\right)$ is $-\widehat{t}_{k} \widehat{t}_{m}(-\mu)$ if $m \neq \infty$, and $-\widehat{t}_{k}(-\mu)$ otherwise, where $\widehat{t}_{j}:=s_{\left|\gamma_{j}\right|,-l_{j}^{J}}=s_{\gamma_{j},-\operatorname{sgn}\left(\gamma_{j}\right) l_{j}^{J}}$. By Proposition 3.22 and (14), we have $\widehat{t}_{k}=s_{\alpha_{p},-M}$ and $\widehat{t}_{m}=s_{\alpha_{p},-(M-1)}$. The rest of the calculation is the same as in the proof mentioned above.

\subsection{Main application}

We summarize the main results in [22], cf. also [20,21]. The setup is that of untwisted affine root systems.

Theorem 3.24 [22] Consider a composition $\mathbf{p}=\left(p_{1}, \ldots, p_{k}\right)$ and the corresponding crystal $B:=\bigotimes_{i=1}^{k} B^{p_{i}, 1}$. Let $\lambda:=\omega_{p_{1}}+\ldots+\omega_{p_{k}}$, and let $\Gamma_{\mathrm{lex}}$ be a corresponding lex $\lambda$-chain (see above).

(1) The (combinatorial) crystal $\mathcal{A}\left(\Gamma_{\mathrm{lex}}\right)$ is isomorphic to the subgraph of $B$ consisting of the dual Demazure arrows, via a specific bijection which preserves the weights of the vertices.

(2) If the vertex $b$ of $B$ corresponds to $J$ under the isomorphism in part (1), then the energy is given by $D_{B}(b)-C=-$ height $(J)$, where $C$ is a global constant.

Remarks 3.25 (1) The entire crystal $B$ is realized in [22] in terms of the so-called quantum LS path model. If we identify the two, the bijection in Theorem 3.24 (1) is the "forgetful map" from the quantum alcove model to the quantum LS path model, so it is a very natural map. Therefore, we think of the former model as a mirror image of the latter, via this bijection. However, if we use this identification to construct the non-dual Demazure arrows in the quantum alcove model, we quickly realize that, in general, the constructions are considerably more involved than (15)-(16), cf. Remark 4.8 (1) and Example 4.9.

(2) Although the quantum alcove model so far misses the non-dual Demazure arrows, it has the advantage of being a discrete model. Therefore, combinatorial methods are applicable, for instance in proving the independence of the model from the choice of an initial alcove path (or $\lambda$-chain of roots), see below, including the application in Remark 3.27 (2). This should be compared with the continuous arguments used for the similar purpose in the Littelmann path model [27].

(3) Theorem 3.24, combined with the Ram-Yip formula for Macdonald polynomials [30], implies that the graded character of a tensor product of column shape KR modules (the grading being by the energy function) concides with the corresponding Macdonald polynomial specialized at $t=0$ [22].

Based on Theorem 3.24 (1), as well as on the realization of the same subgraph of $B$ in types $A$ and $C$ in terms of a different $\lambda$-chain (see Theorems 4.7 and 4.22), we make the following conjecture. 
Conjecture 3.26 Theorem 3.24 holds for any choice of a $\lambda$-chain (instead of a lex $\lambda$-chain).

We plan to prove this conjecture in [19] by using Theorem 3.24 as the starting point. Then, given two $\lambda$-chains $\Gamma$ and $\Gamma^{\prime}$, we would construct a bijection between $\mathcal{A}(\Gamma)$ and $\mathcal{A}\left(\Gamma^{\prime}\right)$ preserving the dual Demazure arrows, the weights of the vertices, and the height statistic; this would mean that the quantum alcove model does not depend on the choice of a $\lambda$-chain. This construction will be based on generalizing to the quantum alcove model the so-called Yang-Baxter moves in [15]. As a result, we would obtain a collection of a priori different affine crystal isomorphisms between $B$ and $\mathcal{A}(\Gamma)$.

Remarks 3.27 (1) We believe that the bijections mentioned above would be identical.

In fact, this would clearly be the case if all the tensor factors of $B$ are perfect crystals, see Sect. 2.1. Indeed, then the subgraph of $B$ consisting of the dual Demazure arrows is connected, so there is no more than one isomorphism between it and $\mathcal{A}(\Gamma)$.

(2) In the case when all the tensor factors of $B$ are perfect crystals, a corollary of the work in [19] would be the following application of the quantum alcove model, cf. Remark 3.27 (1). By making specific choices for the $\lambda$-chains $\Gamma$ and $\Gamma^{\prime}$, the bijection between $\mathcal{A}(\Gamma)$ and $\mathcal{A}\left(\Gamma^{\prime}\right)$ mentioned above would give a uniform realization of the combinatorial $R$-matrix (see Remark $2.10(2)$ ); the details will appear in the forthcoming paper [19]. In fact, we believe that the previous statement would hold in full generality, rather than just the perfect case.

\section{The quantum alcove model in types $A$ and $C$}

In this section we specialize the quantum alcove model to types $A$ and $C$, and prove that the bijections constructed in [18], from the objects of the specialized quantum alcove model to the tensor products of the corresponding KN columns (see Sect. 2.1), are affine crystal isomorphisms.

\subsection{Type $A$}

We start with the basic facts about the root system of type $A_{n-1}$. We can identify the space $\mathfrak{h}_{\mathbb{R}}^{*}$ with the quotient $V:=\mathbb{R}^{n} / \mathbb{R}(1, \ldots, 1)$, where $\mathbb{R}(1, \ldots, 1)$ denotes the subspace in $\mathbb{R}^{n}$ spanned by the vector $(1, \ldots, 1)$. Let $\varepsilon_{1}, \ldots, \varepsilon_{n} \in V$ be the images of the coordinate vectors in $\mathbb{R}^{n}$. The root system is $\Phi=\left\{\alpha_{i j}:=\varepsilon_{i}-\varepsilon_{j}: i \neq\right.$ $j, 1 \leq i, j \leq n\}$. The simple roots are $\alpha_{i}=\alpha_{i, i+1}$, for $i=1, \ldots, n-1$. The highest root $\widetilde{\alpha}=\alpha_{1 n}$. We let $\alpha_{0}=\theta=\alpha_{n 1}$. The weight lattice is $\Lambda=\mathbb{Z}^{n} / \mathbb{Z}(1, \ldots, 1)$. The fundamental weights are $\omega_{i}=\varepsilon_{1}+\ldots+\varepsilon_{i}$, for $i=1, \ldots, n-1$. A dominant weight $\lambda=\lambda_{1} \varepsilon_{1}+\ldots+\lambda_{n-1} \varepsilon_{n-1}$ is identified with the partition $\left(\lambda_{1} \geq \lambda_{2} \geq \ldots \geq \lambda_{n-1} \geq\right.$ $\left.\lambda_{n}=0\right)$ having at most $n-1$ parts. Note that $\rho=(n-1, n-2, \ldots, 0)$. Considering the Young diagram of the dominant weight $\lambda$ as a concatenation of columns, whose heights are $\lambda_{1}^{\prime}, \lambda_{2}^{\prime}, \ldots$, corresponds to expressing $\lambda$ as $\omega_{\lambda_{1}^{\prime}}+\omega_{\lambda_{2}^{\prime}}+\ldots$ (as usual, $\lambda^{\prime}$ is the conjugate partition to $\lambda$ ). 
Fig. 3 The quantum Bruhat graph on $S_{3}$

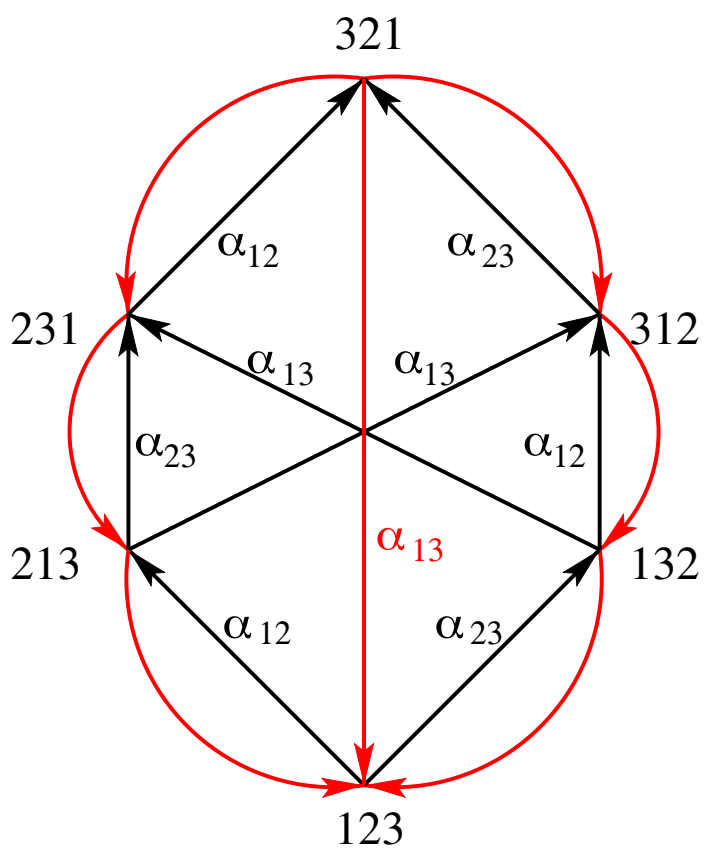

The Weyl group $W$ is the symmetric group $S_{n}$, which acts on $V$ by permuting the coordinate vectors $\varepsilon_{1}, \ldots, \varepsilon_{n}$. Permutations $w \in S_{n}$ are written in one-line notation $w=w(1) \ldots w(n)$. For simplicity, we use the same notation $(i, j)$ with $1 \leq i<j \leq n$ for the root $\alpha_{i j}$ and the reflection $s_{\alpha_{i j}}$, which is the transposition $t_{i j}$ of $i$ and $j$. We recall a criterion for the edges of the type $A$ quantum Bruhat graph. We need the circular order $\prec_{i}$ on $[n]$ starting at $i$, namely $i \prec_{i} i+1 \prec_{i} \ldots \prec_{i} n \prec_{i} 1 \prec_{i} \ldots \prec_{i} i-1$. It is convenient to think of this order in terms of the numbers $1, \ldots, n$ arranged on a circle clockwise. We make the convention that, whenever we write $a \prec b \prec c \prec \ldots$, we refer to the circular order $\prec=\prec_{a}$.

Proposition 4.1 [18] For $1 \leq i<j \leq n$, we have an edge $w \stackrel{(i, j)}{\longrightarrow} w(i, j)$ if and only if there is no $k$ such that $i<k<j$ and $w(i) \prec w(k) \prec w(j)$.

Example 4.2 The quantum Bruhat graph of type $A_{2}$, i.e., on the symmetric group $S_{3}$, is indicated in Fig. 3.

We now consider the specialization of the quantum alcove model to type $A$. For any $k=1, \ldots, n-1$, we have the following $\omega_{k}$-chain, from $A_{\circ}$ to $A_{-\omega_{k}}$, denoted by $\Gamma(k)[23]:$

$$
\begin{array}{llll}
((k, k+1), & (k, k+2) & , \ldots, & (k, n), \\
(k-1, k+1), & (k-1, k+2), \ldots, & (k-1, n), \\
\vdots & \vdots & & \vdots \\
(1, k+1), & (1, k+2) & , \ldots, & (1, n)) .
\end{array}
$$


Example 4.3 We specialize (23) to $n=4$ and $k=1,2,3$. It is best to visualize $\Gamma(k)$ based on a column of height $n$ broken into two pieces, with the top part of height $k$ and the bottom one of height $n-k$; then $\Gamma(k)$ is obtained by pairing row numbers in the top and bottom parts, in the prescribed order.
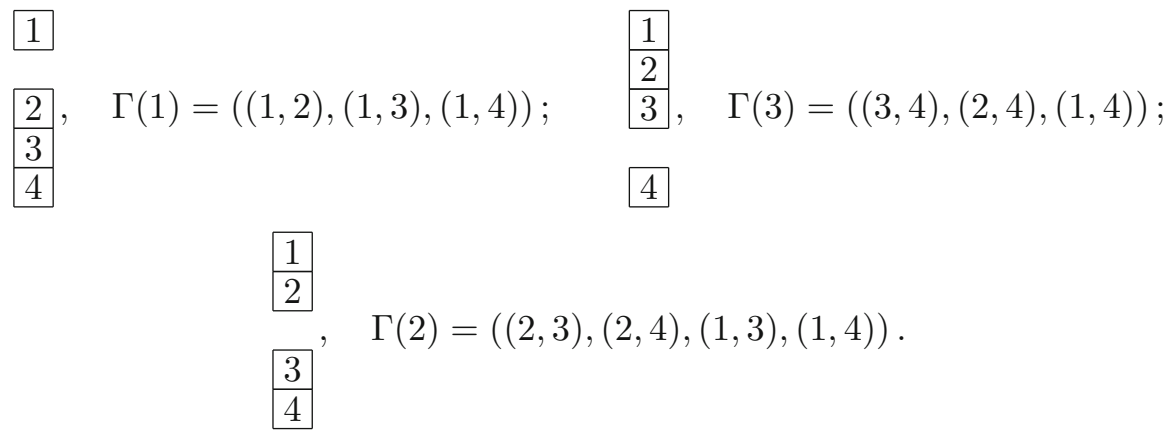

Fix a dominant weight/partition $\lambda$ for the remainder of this section. We construct a $\lambda$ chain $\Gamma=\left(\beta_{1}, \beta_{2}, \ldots, \beta_{m}\right)$ as the concatenation $\Gamma:=\Gamma^{1} \ldots \Gamma^{\lambda_{1}}$, where $\Gamma^{j}=\Gamma\left(\lambda_{j}^{\prime}\right)$. Let $J=\left\{j_{1}<\cdots<j_{s}\right\}$ be a set of folding positions in $\Gamma$, not necessarily admissible, and let $T$ be the corresponding list of roots of $\Gamma$, also viewed as transpositions. The factorization of $\Gamma$ induces a factorization of $T$ as $T=T^{1} T^{2} \ldots T^{\lambda_{1}}$, and of $\Delta=\Gamma(J)$ as $\Delta=\Delta^{1} \ldots \Delta^{\lambda_{1}}$. Recalling that the roots in $\Delta$ were denoted $\gamma_{k}$, we use the notation $\gamma_{k} \in \Delta^{q}$ to indicate that the $k$ th root in $\Delta$ falls in the segment $\Delta^{q}$ (rather than the fact that $\Delta^{q}$ contains a root equal to $\gamma_{k}$ ). We denote by $T^{1} \ldots T^{j}$ the permutation obtained by composing the transpositions in $T^{1}, \ldots, T^{j}$ left to right. For $w \in W$, let $w_{i}=w(i)$. For $w$ written in one-line notation as $w=w_{1} w_{2} \ldots w_{n}$, let $w[i, j]=w_{i} \ldots w_{j}$.

We now recall from [18] the construction of the correspondence between the type $A$ quantum alcove model and model based on diagram fillings.

Definition 4.4 Let $\pi_{j}=\pi_{j}(T):=T^{1} \ldots T^{j}$. We define the filling map, which associates with each $J \subseteq[m]$ a filling of the Young diagram $\lambda$, by

$$
\text { fill }(J)=\operatorname{fill}(T):=C_{1} \ldots C_{\lambda_{1}}, \text { where } C_{i}:=\pi_{i}\left[1, \lambda_{i}^{\prime}\right]
$$

see the notation above. We define the sorted filling map sfill $(J)$ by sorting ascendingly the columns of fill $(J)$.

In other words, the $i$ th column $C_{i}$ of fill $(J)$ consists of the first $\lambda_{i}^{\prime}$ entries of the permutation $\pi_{i}$, written in one-line notation; see Example 4.5.

Example 4.5 Let $n=3$ and $\lambda=(4,3,0)$, which is identified with $4 \varepsilon_{1}+3 \varepsilon_{2}=$

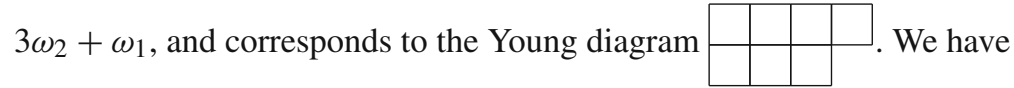

$$
\begin{aligned}
\Gamma= & \Gamma^{1} \Gamma^{2} \Gamma^{3} \Gamma^{4}=\Gamma(2) \Gamma(2) \Gamma(2) \Gamma(1) \\
& =(\underline{(2,3)}, \underline{(1,3)}|\underline{(2,3)},(1,3)| \underline{(2,3)},(1,3) \mid \underline{(1,2)},(1,3)),
\end{aligned}
$$


where we underlined the roots in positions $J=\{1,2,3,5,7\}$. Then

$$
\begin{aligned}
T & =((2,3),(1,3)|(2,3)|(2,3) \mid(1,2)), \text { and } \\
\Gamma(J) & =\Delta \\
& =\Delta^{1} \Delta^{2} \Delta^{3} \Delta^{4}=(\underline{(2,3)}, \underline{(1,2)}|\underline{(3,1)},(2,3)| \underline{(1,3),(2,1) \mid \underline{(2,3)},(3,1)),}
\end{aligned}
$$

where we again underlined the folding positions, and indicated the factorizations of $T$ and $\Delta$ by vertical lines. It is easy to check that $J$ is admissible; indeed, the sequence of permutations (12) corresponding to $J$ is a path in the quantum Bruaht graph, cf. Proposition 4.1 and Example 4.2:

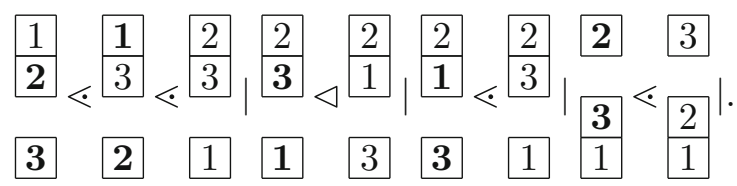

Here each permutation in (12) is written vertically in one-line notation, with the entries in bold to be transposed; moreover, if the transposition to be applied lies in $\Gamma^{i}$, then the corresponding permutation is represented as a broken column with the top part of height $\lambda_{i}^{\prime}$; see the structure of $\Gamma^{i}$ in (23) and Example 4.3. By considering the top part of the last column in each segment and by concatenating these columns left to right, we obtain fill $(J)$, i.e., fill $(J)=$\begin{tabular}{|l|l|l|l|}
\hline 2 & 2 & 2 & 3 \\
\hline 3 & 1 & 3 & \\
\hline
\end{tabular}

Theorem 4.6 [18] [Theorem 4.1] The map sfill is a bijection between $\mathcal{A}(\Gamma)$ and $B^{\otimes \lambda}$, see (8).

We now state the main result of this section.

Theorem 4.7 The map sfill is an affine crystal isomorphism between $\mathcal{A}(\Gamma)$ and the subgraph of $B^{\otimes \lambda}$ consisting of the dual Demazure arrows. In other words, given $\operatorname{sfill}(J)=b$, there is a dual Demazure arrow $b \rightarrow f_{p}(b)$ if and only if $f_{p}(J) \neq \mathbf{0}$, and we have $f_{i}(b)=\operatorname{sfill}\left(f_{i}(J)\right)$.

Remarks 4.8 (1) The affine crystal isomorphism in Theorem 4.7 is unique, cf. Remark 3.27 (1). Therefore, this isomorphism gives the unique way to realize the non-dual Demazure arrows in $\mathcal{A}(\Gamma)$, cf. Remark 3.25 (1) and Example 4.9 below.

(2) In [17] it was proved that the map sfill preserves weights, cf. Lemma 4.10. Furthermore, in [18] it was shown this map translates the height statistic to the charge statistic, which is known to express the energy function in the model based on diagram fillings, cf. Theorem 2.15. This should be compared with Theorem 3.24 (2), where the constant $C$ is 0 in this case.

Example 4.9 In type $A_{2}$, consider $\lambda=(3,2,0)$, the $\lambda$-chain in Example 3.3 , and the admissible subset $J=\{1,2,3,5\}$, cf. Examples 3.6, 3.7, and 3.8. We have 


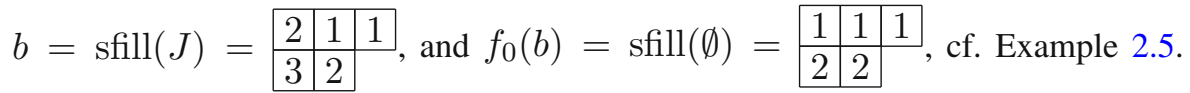

However, $b \rightarrow f_{0}(b)$ is not a dual Demazure arrow, and indeed $f_{0}(J)=\mathbf{0}$, cf. Example 3.8. In order to realize this arrow in the quantum alcove model, we would have to define $f_{0}(J)=\emptyset$. This shows that, in general, the changes in an admissible subset corresponding to non-dual Demazure arrows are hard to control. Nevertheless, such arrows are sometimes still realized by our construction (15), assuming that we drop the corresponding condition $M>1$. For an example, still in type $A_{2}$, consider $\lambda=(3,0,0)$, the $\lambda$-chain $\left(\alpha_{12}, \alpha_{13}, \alpha_{12}, \alpha_{13}, \alpha_{12}, \alpha_{13}\right)$, and $J=\{3,4\}$. We

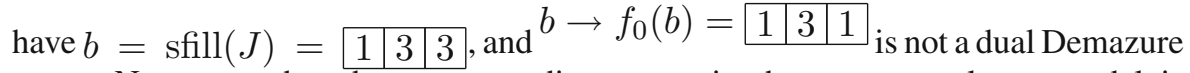
arrow. Now note that the corresponding arrow in the quantum alcove model is $J \mapsto J \cup\{5\}$, which is given by the mentioned relaxed version of (15).

The main idea of the proof of Theorem 4.7 is the following. The signature of a filling, used to define the crystal operator $f_{p}$, can be interpreted as a graph similar to the graph of $g_{\alpha_{p}}$, which is used to define the crystal operator on the corresponding admissible subsequence. The link between the two graphs is given by Lemma 4.12 below, called the height counting lemma, which we now explain.

Let $N_{c}(\sigma)$ denote the number of entries $c$ in a filling $\sigma$. Let $\operatorname{ct}(\sigma)=$ $\left(N_{1}(\sigma), \ldots, N_{n}(\sigma)\right)$ be the content of $\sigma$, which is identified with a type $A_{n-1}$ weight. Let $\sigma[q]$ be the filling consisting of the columns $1,2, \ldots, q$ of $\sigma$. Given a $\lambda$-chain and a corresponding sequence $J$ (not necessarily admissible), recall the related notation, including the heights $l_{k}^{J}$ in (10), the sequence of roots $\Delta$, and its factorization illustrated in (25).

Lemma 4.10 [17] [Proposition 3.6] Let $J \subseteq[m]$, and $\sigma=\operatorname{sfill}(J)$. Then we have $\mu(J)=\operatorname{ct}(\sigma)$.

Corollary 4.11 Let $J \subseteq[m], \sigma=\operatorname{fill}(J)$, and $\alpha \in \Phi$. Then $\operatorname{sgn}(\alpha) l_{\alpha}^{\infty}=$ $\left\langle\operatorname{ct}(\sigma), \alpha^{\vee}\right\rangle$.

The height counting lemma can be viewed as an extension of Corollary 4.11.

Lemma 4.12 [17] [Proposition 4.1] Let $J \subseteq[m]$, and $\sigma=$ fill $(J)$. For a fixed $k$, let $\gamma_{k}=(c, d)$ be a root in $\Delta^{q+1}$. We have

$$
\operatorname{sgn}\left(\gamma_{k}\right) l_{k}^{J}=\left\langle\operatorname{ct}(\sigma[q]), \gamma_{k}^{\vee}\right\rangle=N_{c}(\sigma[q])-N_{d}(\sigma[q]) .
$$

We now introduce notation to be used for the remainder of this section. Let $p \in$ $\{1, \ldots, n-1\}$. Let $J$ be an admissible subset and let $\sigma=\operatorname{sfill}(J)=C_{1} \ldots C_{\lambda_{1}}$. For $i=1, \ldots, \lambda_{1}$, let $a_{i}:=\left\langle\operatorname{ct}\left(C_{i}\right), \alpha_{p}^{\vee}\right\rangle$, and note that $a_{i} \in\{1,-1,0\}$; here $a_{i}=1$ (resp. $a_{i}=-1$ ) corresponds to $C_{i}$ containing $p$ but not $p+1$ (resp. $p+1$ but not $p$ ), while $a_{i}=0$ corresponds to $C_{i}$ containing both $p$ and $p+1$, or neither of them.

The sequence $a_{i}$ corresponds to the $p$-signature from Sect. 2.1, as we now explain. For $j=0, \ldots, \lambda_{1}$, let $h_{j}:=\left\langle\operatorname{ct}(\sigma[j]), \alpha_{p}^{\vee}\right\rangle=\sum_{i=0}^{j} a_{i}$, where we set $a_{0}=h_{0}:=0$. It is useful to think of this sequence as a piecewise linear function, by analogy with the function $g_{\alpha_{p}}$ used to define the crystal operator $f_{p}$ in the quantum alcove model. 

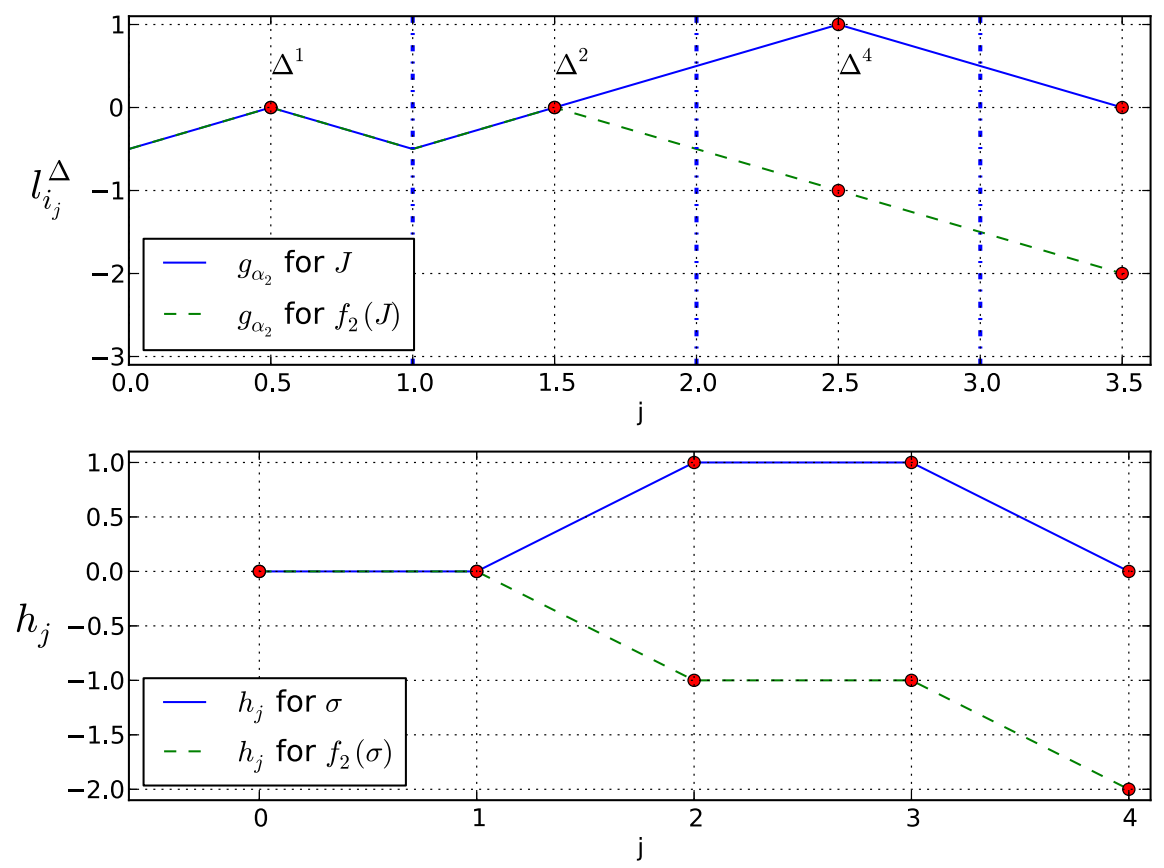

Fig. 4 Effects of $f_{2}$ on $g_{\alpha}$ and $h_{j}$

Let $M^{\prime}$ be the maximum of $h_{j}$, and let $m^{\prime}$ be minimal with the property $h_{m^{\prime}}=M^{\prime}$. We clearly have $M^{\prime} \geq 0$. If $M^{\prime}>0$ then $a_{m^{\prime}}=1$, and $m^{\prime}$ is the position of the rightmost + in the $p$-signature of $\sigma$. It follows that $f_{p}$ changes the $p$ in column $m^{\prime}$ of $\sigma$ to $p+1$. The previous observations hold if we replace $\alpha_{p}, f_{p}$, and the $p$-signature with $\alpha_{0}, f_{0}$, and the 0 -signature, while at the same time we replace the entries $p$ and $p+1$ in a filling with $n$ and 1, respectively. Therefore, from now on we assume that the index $p$ is in $\{0,1, \ldots, n-1\}$.

Example 4.13 We continue Example 4.5. Let $\sigma=\operatorname{sfill}(J)=$\begin{tabular}{|l|l|l|l|}
\hline 2 & 1 & 2 & 3 \\
\hline 3 & 2 & 3
\end{tabular} , then

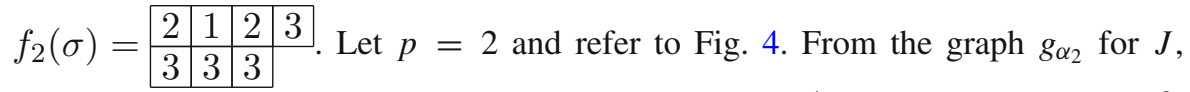
we can see that $M=1$. We note that $m=7$, with $\gamma_{7} \in \Delta^{4}$, and $k=4$ with $\gamma_{k} \in \Delta^{2}$. So $f_{2}(J)=(J \backslash\{7\}) \cup\{4\}=\{1,2,3,4,5\}$, and

$$
\Gamma\left(f_{2}(J)\right)=(\underline{(2,3)}, \underline{(1,2)}|\underline{(3,1)}, \underline{(2,3)}| \underline{(1,2)},(3,1) \mid(3,2),(3,1)),
$$

where we underlined roots in positions $f_{2}(J)$. From the graph corresponding to $h_{j}$ for $J$, we can see that $M^{\prime}=1$ and $m^{\prime}=2$.

Lemma 4.14 If $\alpha_{p}=\gamma_{k} \in \Delta^{q}$ with $k \notin J$ then $a_{q}=1$. 
Proof Recall that $\alpha_{p}=\gamma_{k}=w\left(\beta_{k}\right)$ for the corresponding $w$ defined in (9), and let $\beta_{k}=(b, c)$. The result follows from the claim that $w(b)=\pi_{q}(b)$ and $w(c)=\pi_{q}(c)$ (cf. Definition 4.4), which is a consequence of the structure of $\Gamma^{q}$ (cf. (23)), as we now explain. The only reflections in $\Gamma^{q}$ to the right of $\beta_{k}$ that affect values in positions $b$ or $c$ are $\left(b, c^{\prime}\right)$ for $c^{\prime}>c$, and $\left(b^{\prime}, c\right)$ for $b^{\prime}<b$. Applying these reflections (on the right) to any $w^{\prime}$ satisfying $w^{\prime}(b)=w(b)$ and $w^{\prime}(c)=w(c)$ does not give an edge in the quantum Bruhat graph, by the corresponding criterion in Proposition 4.1.

Fix an admissible subset $J$, and recall Proposition 3.22, including the notation therein. In particular, $M$ is the maximum of $g_{\alpha_{p}}$. Moreover, if $M>\delta_{p, 0}$ we defined $k \notin J$ and $m \in J \cup\{\infty\}$ with $\gamma_{k}=\alpha_{p}, \operatorname{sgn}\left(\alpha_{p}\right) l_{k}^{J}=M-1$, and $\gamma_{m}=\alpha_{p}$ when $m \neq \infty$. We will implicitly use the following observation when applying Lemma 3.11 in the next two proofs: if $a_{i} \neq 0$ then $\operatorname{sgn}\left(a_{i}\right)=\operatorname{sgn}\left(\pi_{i}^{-1}\left(\alpha_{p}\right)\right)$, where $\pi_{i}$ is given in Definition 4.4.

Proposition 4.15 We have $M \geq M^{\prime}$. If $M \geq \delta_{p, 0}$ then $M=M^{\prime}$.

Proof We first prove that $M \geq M^{\prime}$. By Corollary 4.11 we have $h_{\lambda_{1}}=\operatorname{sgn}(\alpha) l_{\alpha_{p}}^{\infty}$, so the case $M^{\prime}=h_{\lambda_{1}}$ is clear. The case $M^{\prime}=0$ is trivial, since $M \geq 0$. Therefore, we can assume that the maximum of the sequence $h_{i}$ does not occur at its endpoints $i=0$ and $i=\lambda_{1}$. Then we can find $i<j$ such that $h_{i}=M^{\prime}, a_{i}>0, a_{j}<0$, and $a_{t}=0$ for $t \in(i, j)$. By Lemma 3.11 there exists $\gamma_{k^{\prime}}=\alpha_{p} \in \Delta^{q}$ with $q \in(i, j]$, and by Lemma 4.12 we have $\operatorname{sgn}\left(\alpha_{p}\right) l_{k^{\prime}}^{J}=h_{q-1}=h_{i}=M^{\prime}$. Hence $M \geq M^{\prime}$.

By (14), Lemma 4.12, and Corollary 4.11, all the values of $g_{\alpha_{p}}$ at points in $\mathbb{Z}+\frac{1}{2}$ are among the values $h_{i}$. If $M \geq \delta_{p, 0}$ then, by Propositions 3.16 and 3.19, the maximum $M$ is attained at a point in $\mathbb{Z}+\frac{1}{2}$. It follows that $M \leq M^{\prime}$, hence $M=M^{\prime}$.

The previous proposition states that $M=M^{\prime}$ except in a few corner cases that occur when $p=0$. We will sometimes use one symbol in favor of the other to allude to the corresponding graph.

Proposition 4.16 Assume that $M>\delta_{p, 0}$, so $M=M^{\prime}$ (by Proposition 4.15) and $f_{p}(J) \neq \mathbf{0}$. Then $\gamma_{k} \in \Delta^{m^{\prime}}$. If $m \neq \infty$, so $\gamma_{m} \in \Delta^{m^{\prime \prime}}$ for some $m^{\prime \prime} \geq m^{\prime}$, then $a_{i}=0$ for $i \in\left(m^{\prime}, m^{\prime \prime}\right)$; if $m=\infty$ then $a_{i}=0$ for $i>m^{\prime}$.

Proof Assuming $\gamma_{k} \in \Delta^{j}$, by Proposition 3.22 (2), Lemma 4.14, and Lemma 4.12, we have $a_{j}=1$ and $\operatorname{sgn}\left(\alpha_{p}\right) l_{k}^{J}=M-1=h_{j-1}$. It follows that $h_{j}=M=M^{\prime}$. By the definition of $m^{\prime}$ and the fact that $M^{\prime}>0$, we have $1 \leq m^{\prime} \leq j$ and $a_{m^{\prime}}=1$. By way of contradiction suppose that $m^{\prime}<j$. It follows that the set $\left\{i \in\left(m^{\prime}, j\right] \mid a_{i} \neq 0\right\}$ is not empty, so let $t$ be its minimum. We have $a_{t}=-1$ and $t<j$, because $a_{t}=1$ would imply $h_{t}>h_{m^{\prime}}=M^{\prime}$. We can now apply Lemma 3.11 to show that there exists $\gamma_{k^{\prime}}=\alpha_{p} \in \Delta^{q}$ with $q \in\left(m^{\prime}, t\right]$ and $k^{\prime} \in J$. By Lemma 4.12, we have $\operatorname{sgn}\left(\alpha_{p}\right) l_{k^{\prime}}^{J}=h_{q-1}=h_{m^{\prime}}=M^{\prime}=M$. Thus, since $k^{\prime}<k<m$, the minimality of $m$ is contradicted. We conclude that $j=m^{\prime}$. If $m \neq \infty$, we can use a similar proof to conclude that the set $\left\{i \in\left(m^{\prime}, m^{\prime \prime}\right) \mid a_{i} \neq 0\right\}$ is empty. The case $m=\infty$ is done similarly. 
Proof of Theorem 4.7 We continue to use the notation from the above setup. Recall that $b=\operatorname{sfill}(J)$. The statement that there is a dual Demazure arrow $b \rightarrow f_{p}(b)$ if and only if $f_{p}(J) \neq \mathbf{0}$ follows from Proposition 4.15; indeed, it is clear that $\varphi_{p}(b)=M^{\prime}$, whereas $\varphi_{p}(J)=M-\delta_{p, 0}$ in the quantum alcove model if $M \geq \delta_{p, 0}$, by Theorem 3.9 (2).

We next show that $f_{p}(b)=\operatorname{sfill}\left(f_{p}(J)\right)$, when $f_{p}(J) \neq \mathbf{0}$. Since $f_{p}(b) \neq \mathbf{0}$, we have $M^{\prime}>0$, and $f_{p}$ changes the $p$ in column $m^{\prime}$ to $p+1\left(f_{0}\right.$ changes $n$ to 1 and sorts the column). Now let us turn to $f_{p}(J)$, where we write the admissible subset $J$ as $\left\{j_{1}<\cdots<j_{s}\right\}$. Let $w_{i}:=r_{j_{1}} \ldots r_{j_{i}}$ be the corresponding sequence of permutations. (Recall that the filling fill $(J)$ is constructed from a subsequence of $w_{i}$, see Definition 4.4.) We assume $m \neq \infty$, as the case $m=\infty$ is proved similarly. There exist $a<b$ such that

$$
j_{a}<k<j_{a+1}<\cdots<j_{b}=m<j_{b+1}
$$

if $a=0$ or $b=s$, then the corresponding indices $j_{a}$, respectively $j_{b+1}$ are missing. The sequence of permutations associated to $f_{p}(J)$ is

$$
1, w_{1}, \ldots, w_{a}, s_{p} w_{a}, s_{p} w_{a+1}, \ldots, s_{p} w_{b-1}=w_{b}, w_{b+1}, \ldots, w_{s}
$$

(see (19)). By the first part of Proposition 4.16 and by using the notation therein, we conclude that sfill $\left(f_{p}(J)\right)$ is obtained from sfill $(J)$ by interchanging $p$ and $p+1$ in columns $i$ for $i \in\left[m^{\prime}, m^{\prime \prime}\right.$ ) (interchange $n$ with 1 if $p=0$ ). By the second part of Proposition 4.16, this amounts to changing the $p$ in column $m^{\prime}$ to $p+1$ ( $n$ to 1 if $p=0)$.

\subsection{Type $C$}

We start with the basic facts about the root system of type $C_{n}$. We can identify the space $\mathfrak{h}_{\mathbb{R}}^{*}$ with $V:=\mathbb{R}^{n}$, the coordinate vectors being $\varepsilon_{1}, \ldots, \varepsilon_{n}$. The root system is $\Phi=\left\{ \pm \varepsilon_{i} \pm \varepsilon_{j}: 1 \leq i<j \leq n\right\} \cup\left\{ \pm 2 \varepsilon_{i}: 1 \leq i \leq n\right\}$. The simple roots are $\alpha_{i}=\varepsilon_{i}-\varepsilon_{i+1}$, for $i=1, \ldots, n-1$, and $\alpha_{n}=2 \varepsilon_{n}$. The highest root $\widetilde{\alpha}=2 \varepsilon_{1}$. We let $\alpha_{0}=\theta=-2 \varepsilon_{1}$. The weight lattice is $\Lambda=\mathbb{Z}^{n}$. The fundamental weights are $\omega_{i}=\varepsilon_{1}+\cdots+\varepsilon_{i}$, for $i=1, \ldots, n$. A dominant weight $\lambda=\lambda_{1} \varepsilon_{1}+\cdots+\lambda_{n} \varepsilon_{n}$ is identified with the partition $\left(\lambda_{1} \geq \lambda_{2} \geq \cdots \geq \lambda_{n-1} \geq \lambda_{n} \geq 0\right)$ of length at most $n$. Note that $\rho=(n, n-1, \ldots, 1)$. Like in type $A$, writing the dominant weight $\lambda$ as a sum of fundamental weights corresponds to considering the Young diagram of $\lambda$ as a concatenation of columns. We fix a dominant weight $\lambda$ throughout this section.

The Weyl group $W$ is the group of signed permutations $B_{n}$, which acts on $V$ by permuting the coordinates and changing their signs. A signed permutation is a bijection $w$ from $[\bar{n}]:=\{1<2<\cdots<n<\bar{n}<\overline{n-1}<\cdots<\overline{1}\}$ to $[\bar{n}]$ satisfying $w(\bar{l})=\overline{w(i)}$. Here $\bar{l}$ is viewed as $-i$, so $\overline{\bar{l}}=i,|\bar{l}|=i$, and $\operatorname{sign}(\bar{l})=$ -1 . We use both the window notation $w=w_{1} \ldots w_{n}$ and the full one-line notation $w=w(1) \ldots w(n) w(\bar{n}) \ldots w(\overline{1})$ for signed permutations. For simplicity, given $1 \leq$ $i<j \leq n$, we denote by $(i, j)$ the root $\varepsilon_{i}-\varepsilon_{j}$ and the corresponding reflection, 
which is identified with the composition of transpositions $t_{i j} t_{\overline{\jmath l}}$. Similarly, we denote by $(i, \bar{J})=(j, \bar{l})$, for $1 \leq i<j \leq n$, the root $\varepsilon_{i}+\varepsilon_{j}$ and the corresponding reflection, which is identified with the composition of transpositions $t_{i \bar{j}} t_{j \bar{l}}$. Finally, we denote by $(i, \bar{l})$ the root $2 \varepsilon_{i}$ and the corresponding reflection, which is identified with the transposition $t_{i \bar{\imath}}$.

We recall a criterion for the edges of the type $C$ quantum Bruhat graph. We need the circular order $\prec_{i}$ on $[\bar{n}]$ starting at $i$, which is defined in the obvious way, cf. Sect. 4.1. It is convenient to think of this order in terms of the numbers $1, \ldots, n, \bar{n}, \ldots, \overline{1}$ arranged on a circle clockwise. We make the same convention as in Sect. 4.1 that, whenever we write $a \prec b \prec c \prec \ldots$, we refer to the circular order $\prec=\prec_{a}$.

\section{Proposition 4.17 [18]}

(1) Given $1 \leq i<j \leq n$, we have an edge $w \stackrel{(i, j)}{\longrightarrow} w(i, j)$ if and only if there is no $k$ such that $i<k<j$ and $w(i) \prec w(k) \prec w(j)$.

(2) Given $1 \leq i<j \leq n$, we have an edge $w \stackrel{(i, \bar{J})}{\longrightarrow}$ if and only if $w(i)<$ $w(\bar{J}), \operatorname{sign}(w(i))=\operatorname{sign}(w(\bar{J}))$, and there is no $k$ such that $i<k<\bar{J}$ and $w(i)<w(k)<w(\bar{J})$.

(3) Given $1 \leq i \leq n$, we have an edge $w \stackrel{(i, \bar{l})}{\longrightarrow} w(i, \bar{l})$ if and only if there is no $k$ such that $i<k<\bar{l}$ (or, equivalently, $i<k \leq n$ ) and $w(i) \prec w(k) \prec w(\bar{l})$.

We now consider the specialization of the quantum alcove model to type $C$. For any $k=1, \ldots, n$, we have the following $\omega_{k}$-chain, from $A_{\circ}$ to $A_{-\omega_{k}}$, denoted by $\Gamma(k)$ [16]:

$$
\begin{aligned}
\Gamma(k):= & \Gamma_{l}(k) \Gamma_{r}(k) \text { where } \\
\Gamma_{l}(k):= & \Gamma_{k k} \ldots \Gamma_{k 1}, \quad \Gamma_{r}(k):=\Gamma_{k} \ldots \Gamma_{2}, \\
\Gamma_{i}:= & ((i, \overline{i-1}),(i, \overline{i-2}), \ldots,(i, \overline{1})), \\
\Gamma_{k i}:= & ((i, k+1),(i, k+2), \ldots,(i, n), \\
& (i, \bar{l}), \\
& (i, \bar{n}), \quad(i, \overline{n-1}), \ldots,(i, \overline{k+1}), \\
& (i, \overline{i-1}),(i, \overline{i-2}), \ldots,(i, \overline{1})) .
\end{aligned}
$$

Example 4.18 We specialize (27) to $n=3$ and $k=2$. In this case

$$
\Gamma(2)=\Gamma_{l}(2) \Gamma_{r}(2)=((2,3),(2, \overline{2}),(2, \overline{3}),(2, \overline{1}),(1,3),(1, \overline{1}),(1, \overline{3}) \mid(2, \overline{1})) .
$$

On the right hand side we use a vertical line to denote the end of $\Gamma_{l}(2)$. For a graphical interpretation similar to the one in Example 4.3, based on broken columns, we refer to [18][eq. (7.1)].

Fix a dominant weight/partition $\lambda$ for the remainder of this section. We construct a $\lambda$-chain $\Gamma=\left(\beta_{1}, \beta_{2}, \ldots, \beta_{m}\right)$ as a concatenation $\Gamma:=\Gamma^{1} \ldots \Gamma^{\lambda_{1}}$, where $\Gamma^{j}=$ $\Gamma\left(\lambda_{j}^{\prime}\right)$; we also let $\Gamma_{l}^{j}:=\Gamma_{l}\left(\lambda_{j}^{\prime}\right)$ and $\Gamma_{r}^{j}:=\Gamma_{r}\left(\lambda_{j}^{\prime}\right)$. Like in type $A$, given a set $J=\left\{j_{1}<\cdots<j_{s}\right\}$ of folding positions in $\Gamma$, not necessarily admissible, we let $T$ be 
the corresponding list of roots of $\Gamma$, also viewed as type $C$ transpositions. We factor $\Gamma$ as $\Gamma=\widetilde{\Gamma}^{1} \ldots \widetilde{\Gamma}^{2 \lambda_{1}}$, where $\widetilde{\Gamma}^{2 i-1}=\Gamma_{l}^{i}$ and $\widetilde{\Gamma}^{2 i}=\Gamma_{r}^{i}$, for $1 \leq i \leq \lambda_{1}$. This factorization of $\Gamma$ induces a factorization of $T$ as $T^{1} T^{2} \ldots T^{2 \lambda_{1}}$, and of $\Delta=\Gamma(J)$ as $\Delta=\Delta^{1} \ldots \Delta^{2 \lambda_{1}}$. Like in type $A$, we use the notation $\gamma_{k} \in \Delta^{q}$ to indicate that the $k$ th root in $\Delta$ falls in the segment $\Delta^{q}$. We denote by $T^{1} T^{2} \ldots T^{j}$ the signed permutation obtained by composing the type $C$ transpositions in $T^{1}, \ldots, T^{j}$ left to right. For $w \in W$, let $w_{i}=w(i)$. For $w$ written in the window notation as $w=w_{1} w_{2} \ldots w_{n}$, let $w[i, j]=w_{i} \ldots w_{j}$.

We now recall from [18] the construction of the correspondence between the type $C$ quantum alcove model and model based on diagram fillings; it is useful to compare with Definition 4.4 in type $A$.

Definition 4.19 Let $\pi_{j}=\pi_{j}(T):=T^{1} \ldots T^{j}$. We define the filling map, which associates with each $J \subseteq[\mathrm{m}]$ a filling of the Young diagram $2 \lambda$, by

$$
\text { fill }(J)=\operatorname{fill}(T):=C_{1} \ldots C_{2 \lambda_{1}}, \text { where } C_{i}:=\pi_{i}\left[1, \lambda_{\left\lceil\frac{i}{2}\right\rceil}^{\prime}\right]
$$

see the notation above. We define the sorted filling map sfill $(J)$ by sorting ascendingly the columns of the filling fill $(J)$.

Example 4.20 Let $n=3$ and $\lambda=(2,1,0)$, which is identified with $2 \varepsilon_{1}+\varepsilon_{2}=\omega_{2}+\omega_{1}$ and corresponds to the Young diagram $\square$. We have the following $\lambda$-chain, see Example 4.18:

$$
\begin{aligned}
\Gamma & =\Gamma(2) \Gamma(1)=\Gamma_{l}(2) \Gamma_{r}(2) \Gamma_{l}(1) \Gamma_{r}(1) \\
& =(\underline{(2,3)}, \underline{(2, \overline{2})}, \underline{(2, \overline{3})},(2, \overline{1}),(1,3),(1, \overline{1}),(1, \overline{3})|\underline{(2, \overline{1})} \| \underline{(1,2)},(1,3), \underline{(1, \overline{1})},(1, \overline{3}),(1, \overline{2})|),
\end{aligned}
$$

where we underlined the roots in positions $J=\{1,2,3,8,9,11\}$. On the right hand side we used double vertical lines to indicate the end of $\Gamma(2)$, and single vertical lines indicate the end of $\Gamma_{l}(k)$. Then

$$
T=((2,3),(2, \overline{2}),(2, \overline{3})|(2, \overline{1}) \|(1,2),(1, \overline{1})|)
$$

and

$$
\begin{aligned}
\Gamma(J) & =\Delta=\Delta^{1} \Delta^{2} \Delta^{3} \Delta^{4} \\
& =(\underline{(2,3)}, \underline{(3, \overline{3})}, \underline{(\overline{3}, \overline{2})},(\overline{2}, \overline{1}),(1,3),(1, \overline{1}),(1, \overline{3})|\underline{(\overline{2}, \overline{1})} \| \underline{(2, \overline{1})},(\overline{1}, 3), \underline{(\overline{1}, 1)},(1, \overline{3}),(1, \overline{2})|) ;
\end{aligned}
$$

here we again underlined the folding positions, and indicated the factorizations of $T$ and $\Delta$ by vertical lines. 
It is easy to check that $J$ is admissible using Proposition 4.17; indeed, the sequence of signed permutations (12) corresponding to $J$ is a path in the quantum Bruhat graph:

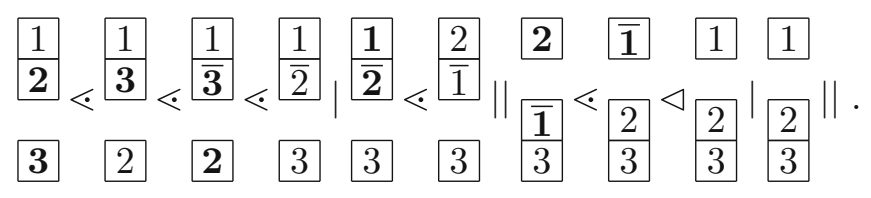

Here, like in Example 4.5 in type $A$, each signed permutation in (12) is written vertically in the window notation, with the entries in bold to be changed by the corresponding reflection; moreover, if this reflection lies in $\Gamma_{l}^{i}$ or $\Gamma_{r}^{i}$, then the corresponding signed permutation is represented as a broken column with the top part of height $\lambda_{i}^{\prime}$; see the structure of $\Gamma_{l}^{i}$ and $\Gamma_{r}^{i}$ in (27) and Example 4.18. By considering the top part of the last column in each segment and by concatenating these columns left to right, we obtain fill $(J)$, i.e., $\operatorname{sfill}(J)=\operatorname{fill}(J)=$\begin{tabular}{|l|l|l|l|}
\hline$\frac{1}{2}$ & 2 & 1 & 1 \\
\hline$\overline{2}$ & $\overline{1}$ &
\end{tabular}.

Recall from (8) the definition of $B^{\otimes \lambda}$, which is now realized with split $\mathrm{KN}$ columns, see Sect. 2.1. As such, its arrows are given by $f_{p}^{2}$, according to Proposition 2.9 (2).

Theorem 4.21 [18] [Theorem 6.1] The map sfill is a bijection between $\mathcal{A}(\Gamma)$ and $B^{\otimes \lambda}$.

We now state the main result of this section, cf. Theorem 4.7 in type $A$.

Theorem 4.22 The map sfill is an affine crystal isomorphism between $\mathcal{A}(\Gamma)$ and the subgraph of $B^{\otimes \lambda}$ consisting of the dual Demazure arrows.

Remarks 4.23 (1) The affine crystal isomorphism in Theorem 4.22 is not guaranteed to be unique in general. However, we believe that it coincides with the bijection that we plan to construct (in a type-independent setup) in order to prove Conjecture 3.26. See Remark 3.27 (1).

(2) In [16] it was proved that the map sfill preserves weights, cf. Lemma 4.24. Furthermore, in [18] it was shown that this map translates the height statistic to the charge statistic, which is known to express the energy function in the model based on KN columns, cf. Theorem 2.15. This should be compared with Theorem 3.24 (2), where the constant $C$ is 0 in this case.

The proof of Theorem 4.21 is parallel to the proof of Theorem 4.7. In this case, we use the height counting lemma in type $C_{n}$, namely Lemma 4.26. As before let $N_{i}(\sigma)$ denote the number of entries $i$ in a filling $\sigma$. Let $c_{i}=c_{i}(\sigma):=\frac{1}{2}\left(N_{i}(\sigma)-N_{\bar{l}}(\sigma)\right)$ and define the content of a filling $\sigma$ as $\operatorname{ct}(\sigma):=\left(c_{1}, c_{2}, \ldots, c_{n}\right)$, which is identified with a type $C_{n}$ weight. Let $\sigma[q]$ be the filling consisting of the columns $1,2, \ldots, q$ of $\sigma$. Given a $\lambda$-chain and a corresponding sequence $J$ (not necessarily admissible), recall the related notation, including the heights $l_{k}^{J}$ in (10), the sequence of roots $\Delta$, and its factorization. 
Lemma 4.24 [16][Proposition 4.6(2)] Let $J \subseteq[m]$, and $\sigma=\operatorname{sfill}(J)$. Then we have $\mu(J)=\operatorname{ct}(\sigma)$.

Corollary 4.25 Let $J \subseteq[m], \sigma=\operatorname{fill}(J)$, and $\alpha \in \Phi$. Then $\operatorname{sgn}(\alpha) l_{\alpha}^{\infty}=$ $\left\langle\operatorname{ct}(\sigma), \alpha^{\vee}\right\rangle$.

Lemma 4.26 [16][Proposition 6.1] Let $J \subseteq[m]$, and $\sigma=$ fill $(J)$. For a fixed $k$, let $\gamma_{k}$ be a root in $\Delta^{q+1}$. We have

$$
\operatorname{sgn}\left(\gamma_{k}\right) l_{k}^{J}=\left\langle\operatorname{ct}(\sigma[q]), \gamma_{k}^{\vee}\right\rangle
$$

We now introduce notation to be used for the remainder of this section. Let $p \in$ $\{0,1, \ldots, n\}$. Let $J$ be an admissible subset, and $\sigma=\operatorname{sfill}(J)=C_{1} \ldots C_{2 \lambda_{1}}$, which is guaranteed to be in $B^{\otimes \lambda}$ by Theorem 4.21. Let $a_{i}:=\left\langle\operatorname{ct}\left(C_{i}\right), \alpha_{p}^{\vee}\right\rangle$. We have $a_{i} \in$ $\left\{-1,-\frac{1}{2}, 0, \frac{1}{2}, 1\right\}$, for $1 \leq p \leq n-1$, and $a_{i} \in\left\{-\frac{1}{2}, 0, \frac{1}{2}\right\}$ for $p \in\{0, n\}$.

Remarks 4.27 (1) The value of $a_{i}$ indicates which entries related to the action of $f_{p}$ are contained in column $C_{i}$, as we now explain. Assuming first that $1 \leq p \leq n-1$, the relevant entries are $\mathcal{P}:=\{p, p+1, \overline{p+1}, \bar{p}\}$. If $a_{i}=1$ (resp. $a_{i}=-1$ ), then $C_{i}$ contains both $p$ and $\overline{p+1}$ (resp. $p+1$ and $\bar{p}$ ). If $a_{i}=\frac{1}{2}\left(\right.$ resp. $\left.a_{i}=-\frac{1}{2}\right)$, then $C_{i}$ contains only one of $p, \overline{p+1}$ (resp. only one of $p+1, \bar{p}$ ), while if $a_{i}=0$ then $C_{i}$ contains both $p$ and $p+1$, or both $\overline{p+1}$ and $\bar{p}$, or none of these elements. For $p=n$, the relevant entries are $n$ and $\bar{n}$. If $a_{i}=\frac{1}{2}$ (resp. $a_{i}=-\frac{1}{2}$ ), then $C_{i}$ contains $n$ (resp. $\bar{n}$ ), while if $a_{i}=0$ then $C_{i}$ contains both $n$ and $\bar{n}$, or none of these elements. The case $p=0$ is similar to $p=n$ : just replace $n$ and $\bar{n}$ with $\overline{1}$ and 1 , respectively.

(2) The sequence $a_{i}$ corresponds to the $p$-signature of the filling $\sigma$. To be more precise, associate with this sequence a $(+,-)$-word by replacing $\pm \frac{1}{2}$ with \pm and \pm 1 with \pm \pm (the 0 's are ignored). If $1 \leq p \leq n-1$, this is the same as the $(+,-)$-word associated with $\sigma$ (see Sect. 2.1) after canceling -+ pairs corresponding to the entries $p$ and $p+1$ ( or $\overline{p+1}$ and $\bar{p}$ ) in a column; similarly for $p=n$ and $p=0$.

Let $h_{j}:=\left\langle\operatorname{ct}(\sigma[j]), \alpha_{p}^{\vee}\right\rangle=\sum_{i=0}^{j} a_{i}$, with $a_{0}=h_{0}:=0$. Like in type $A$, let $M^{\prime} \geq 0$ be the maximum of $h_{j}$, and let $m^{\prime}$ be minimal with the property $h_{m^{\prime}}=M^{\prime}$. If $M^{\prime}>0$ then $m^{\prime}$ is the number of the column containing the entry changed by $f_{p}$. Recall that we need to apply $f_{p}$ twice; the way in which this can happen is described below.

Proposition 4.28 If $1 \leq p \leq n-1$, then we always have one of the following cases related to the action of $f_{p}^{2}$ on the filling $\sigma$.

(i) $m^{\prime}=2 i-1$ and $a_{m^{\prime}}=1: p$ and $\overline{p+1}$ in column $m^{\prime}$ are changed to $p+1$ and $\bar{p}$.

(ii) same as (i) with $m^{\prime}=2 i$.

(iii) $m^{\prime}=2 i$ and $a_{m^{\prime}}=a_{m^{\prime}-1}=\frac{1}{2}$ : columns $m^{\prime}$ and $m^{\prime}-1$ both contain an entry $p$ (or both contain $\overline{p+1})$, and these entries are changed to $p+1$ (resp. $\bar{p})$.

If $p=n$ or $p=0$, then the analog of case (iii) always holds, with $n$ changed to $\bar{n}$, resp. $\overline{1}$ changed to 1 . 
Proof We implicitly use the following observation, which is immediate from the construction of the splitting $(l C, r C)$ of a column $C$ in Definition 2.7: given $x \in[n]$, the column $l C$ contains $x$ or $\bar{x}$ if and only if $r C$ does.

We consider only $1 \leq p \leq n-1$, as the proof is simpler for $p=n$ and $p=0$. We first prove the following claim: if $a_{2 i-1}= \pm \frac{1}{2}$ ( or $a_{2 i}= \pm \frac{1}{2}$ ), then $C_{2 i-1}$ and $C_{2 i}$ contain a single element in $\mathcal{P}$, the two elements have the same absolute value, and the pair $\left(a_{2 i-1}, a_{2 i}\right)$ can take only the following values: $\left(\frac{1}{2}, \frac{1}{2}\right),\left(-\frac{1}{2},-\frac{1}{2}\right)$, or $\left(-\frac{1}{2}, \frac{1}{2}\right)$.

We consider only the case $a_{2 i-1}=\frac{1}{2}$, as the others are completely similar. The assumption implies that column $C_{2 i-1}$ contains a single element in $\mathcal{P}$, namely $p$ or $\overline{p+1}$. In the first case, it is clear that $C_{2 i}$ does not contain $p+1$ or $\overline{p+1}$, but it contains either $p$ or $\bar{p}$. It suffices to rule out the occurence of $\bar{p}$. Assuming it, we deduce that the column $C$ whose splitting is $\left(C_{2 i-1}, C_{2 i}\right)$ contains $z_{j}>p$ and $\overline{z_{j}}$, and the corresponding $t_{j}<z_{j}$ is $p$ (cf. Definition 2.7). But $C$ cannot contain $p+1$ or $\overline{p+1}$, so $z_{j}>p+1$, and the maximality of $t_{j}$ is contradicted. In the second case, we need to rule out the occurence of $p+1$ in $C_{2 i}$. Assuming it, we deduce that $C$ contains $z_{j}=p+1$ and $\overline{z_{j}}$, and we have $t_{j}<p$, so again the maximality of $t_{j}$ is contradicted.

Now consider the $(+,-)$-word associated with the sequence $a_{j}$, see Remark 4.27 (2). Cancel pairs -+ corresponding to the case $\left(a_{2 i-1}, a_{2 i}\right)=\left(-\frac{1}{2}, \frac{1}{2}\right)$ mentioned above. The above claim implies that the resulting word is a concatenation of pairs ++ and -- which come from $a_{j}= \pm 1$ and $\left(a_{2 i-1}, a_{2 i}\right)=\left( \pm \frac{1}{2}, \pm \frac{1}{2}\right)$; recall that for the latter pairs, the claim also gives the corresponding entries in $\mathcal{P}$. The statement of the proposition now follows.

The following is the analog of Lemma 4.14.

Lemma 4.29 If $\alpha_{p}=\gamma_{k} \in \Delta^{2 i-1}$ with $k \notin J$ then we have either $a_{2 i-1}=\frac{1}{2}$ and $a_{2 i}=\frac{1}{2}$, or $a_{2 i-1}=1$. If $\alpha_{p}=\gamma_{k} \in \Delta^{2 i}$ with $k \notin J$ then $a_{2 i}=1$.

Proof We only consider the case corresponding to $\alpha_{p} \in \Delta^{2 i-1}$ and $1 \leq p \leq n-1$, as the others are simpler. We use freely the structure of the chain of roots $\widetilde{\Gamma}^{2 \bar{i}-1}$, see (27). Recall that $\alpha_{p}=\gamma_{k}=w\left(\beta_{k}\right)$ for the corresponding $w$ defined in (9). We have the following cases:

(1) $\beta_{k}=(b, c)$ with $b \leq \lambda_{i}^{\prime}, c>\lambda_{i}^{\prime}$, and $w(b)=p, w(c)=p+1$ (or $w(b)=$ $\overline{p+1}, w(c)=\bar{p})$

(2) $\beta_{k}=(b, \bar{c})$ with $b \leq \lambda_{i}^{\prime}, c>\lambda_{i}^{\prime}$, and $w(b)=p, w(c)=\overline{p+1}$ (or $w(b)=$ $\overline{p+1}, w(c)=p)$

(3) $\beta_{k}=(b, \bar{c})$ with $c<b \leq \lambda_{i}^{\prime}$, and $w(b)=p, w(c)=\overline{p+1}$ (or $w(b)=$ $\overline{p+1}, w(c)=p)$.

We will only consider the first case with $w(b)=p, w(c)=p+1$, as the others are completely similar. We first claim that $w(b)=\pi_{2 i-1}(b)$ (cf. Definition 4.4), i.e., the entry $p$ is not moved by the reflections $s_{\beta_{k^{\prime}}}$ for $\beta_{k^{\prime}}$ in $\widetilde{\Gamma}^{2 i-1}$ with $k^{\prime}>k$. These reflections are $\left(b, c^{\prime}\right)$ with $c^{\prime}>c,(b, \bar{b}),\left(b, \overline{c^{\prime}}\right)$ with $c^{\prime}>\lambda_{i}^{\prime}$, and $\left(b, \overline{c^{\prime}}\right)$ with $c^{\prime}<b$. So if the claim failed, the quantum Bruhat graph criterion in Proposition 4.17 would be violated, because the entry $p+1$ is still in position $c$ when these reflections are applied. 
Let us now track the entry $p+1$ as we apply the subsequent reflections not involving position $b$, by freely using the quantum Bruhat graph criterion. If this entry is not moved by any of these reflections, then $\pi_{2 i-1}(c)=p+1$, so $a_{2 i-1}=\frac{1}{2}$. The first reflection which can move $p+1$ is of the form $\left(b^{\prime}, \bar{c}\right)$ with $b^{\prime}<b$, which means that in position $b^{\prime}$ we will now have the entry $\overline{p+1}$. If this entry is not moved by any of the subsequent reflections $\left(b^{\prime}, \overline{c^{\prime}}\right)$ with $\lambda_{i}^{\prime}<c^{\prime}<c$, then it is not moved by any of the remaining reflections either, so $\pi_{2 i-1}\left(b^{\prime}\right)=\overline{p+1}$, and $a_{2 i-1}=1$. Otherwise, we will have the entry $p+1$ in a position $c^{\prime}$ with $\lambda_{i}^{\prime}<c^{\prime}<c$, and the above reasoning can be applied again (a finite number of times).

Finally, the fact that if $a_{2 i-1}=\frac{1}{2}$ then $a_{2 i}=\frac{1}{2}$ was deduced in the proof of Proposition 4.28.

Recall Proposition 3.22 and the notation therein. $M$ is the maximum of $g_{\alpha_{p}}$, and suppose $M>\delta_{p, 0}$; then $\gamma_{k}=\alpha_{p}$ with $k \notin J, \operatorname{sgn}\left(\alpha_{p}\right) l_{k}^{J}=M-1$, and if $m \neq \infty$ then $\gamma_{m}=\alpha_{p}$ with $m \in J$. The following result is the analog of Proposition 4.15, and its proof is identical. Indeed, the following key fact is still true: if $a_{i} \neq 0$ then $\operatorname{sgn}\left(a_{i}\right)=\operatorname{sgn}\left(\pi_{i}^{-1}\left(\alpha_{p}\right)\right)$, where $\pi_{i}$ is given in Definition 4.19 (simply note that $a_{i}=\left\langle\pi_{i}\left(\omega_{k}\right), \alpha_{p}^{\vee}\right\rangle=\left\langle\omega_{k}, \pi_{i}^{-1}\left(\alpha_{p}^{\vee}\right)\right\rangle$, where $\left.k=\lambda_{\left\lceil\frac{i}{2}\right\rceil}^{\prime}\right)$. This will be needed in the proof of Proposition 4.31 as well.

Proposition 4.30 We have $M \geq M^{\prime}$. If $M \geq \delta_{p, 0}$, then $M=M^{\prime}$.

The following result is the analog of Proposition 4.16.

Proposition 4.31 Assume that $M>\delta_{p, 0}$, so $M=M^{\prime}$ (by Proposition 4.30) and $f_{p}(J) \neq \mathbf{0}$. If $a_{m^{\prime}}=1$ then $\gamma_{k} \in \Delta^{m^{\prime}}$, otherwise $\gamma_{k} \in \Delta^{m^{\prime}-1}$. If $m \neq \infty$, so $\gamma_{m} \in \Delta^{m^{\prime \prime}}$, then $a_{i}=0$ for $i \in\left(m^{\prime}, m^{\prime \prime}\right)$; if $m=\infty$, then $a_{i}=0$ for $i>m^{\prime}$.

Proof Assuming $\gamma_{k} \in \Delta^{j}$, by Proposition 3.22 (2), Lemma 4.29, and Lemma 4.26, we have $\operatorname{sgn}\left(\alpha_{p}\right) l_{k}^{J}=M-1=h_{j-1}$, and either $a_{j}=1$ or $a_{j}=a_{j+1}=\frac{1}{2}$. In the first case, the rest of the proof is essentially identical to that of Proposition 4.16; in particular, we show that $j=m^{\prime}$. In the second case, we have $j=2 i-1$, so it follows that $h_{2 i}=M=M^{\prime}$. Once again, essentially the same proof as that of Proposition 4.16 applies; in particular, we show that $2 i=m^{\prime}$. Note that in both situations we implicitly used the cases in Proposition 4.28.

Proof of Theorem 4.22 The proof is similar to that of Theorem 4.7, so we only point out the extra complexity in type $C$. This has to do with showing that, if $b=\operatorname{sfill}(J)$ and $f_{p}(J) \neq \mathbf{0}$, then $f_{p}^{2}(b)=\operatorname{sfill}\left(f_{p}(J)\right)$. We continue to use the notation from the above setup, and we consider only the case $1 \leq p \leq n-1$, as the others are simpler.

Since $f_{p}(b) \neq \mathbf{0}$, we have $M^{\prime}>0$, and $f_{p}^{2}$ acts on $b$ in one of the ways indicated in Proposition 4.28, cases (i)-(iii). Now let us turn to $f_{p}(J)$, and use the same setup as in the proof of Theorem 4.7, to which we refer. By the first part of Proposition 4.31, we conclude that $\operatorname{sfill}\left(f_{p}(J)\right)$ is obtained from sfill $(J)$ by applying $s_{p}$ to columns $i$ for $i \in\left[m^{\prime}, m^{\prime \prime}\right)$ in cases (i)-(ii), resp. $i \in\left[m^{\prime}-1, m^{\prime \prime}\right)$ in case (iii). By the second part of Proposition 4.31, this amounts to applying $s_{p}$ to column $m^{\prime}$, resp. to columns $m^{\prime}-1$ 
and $m^{\prime}$. By Proposition 4.28, this is the same as the action of $f_{p}^{2}$ on $\operatorname{sfill}(J)$, which concludes the proof. Note that in the above reasoning we implicitly used Remark 4.27 (1).

Acknowledgments Both authors were partially supported by the NSF grant DMS-1101264. The first author gratefully acknowledges the hospitality and support of the Max-Planck-Institut für Mathematik in Bonn, where part of this work was carried out.

\section{References}

1. Briggs, C., Lenart, C.: A charge statistic in type $B$. In preparation

2. Brenti, F., Fomin, S., Postnikov, A.: Mixed bruhat operators and yang-baxter equations for Weyl groups. Int. Math. Res. Not. 8, 419-441 (1999)

3. Fourier, G., Littelmann, P.: Tensor product structure of affine Demazure modules and limit constructions. Nagoya Math. J. 182, 171-198 (2006)

4. Fourier, G., Schilling, A., Shimozono, M.: Demazure structure inside Kirillov-Reshetikhin crystals. J. Algebra 309, 386-404 (2007)

5. Fulton, W.: Young Tableaux. Cambridge University Press, Cambridge (1997)

6. Fulton, W., Woodward, C.: On the quantum product of Schubert classes. J. Algebraic Geom. 13, 641-661 (2004)

7. Gaussent, S., Littelmann, P.: LS-galleries, the path model and MV-cycles. Duke Math. J. 127, 35-88 (2005)

8. Hatayama, G., Kuniba, A., Okado, M., Takagi, T., Yamada, Y.: Remarks on fermionic formula. Recent Developments in Quantum Affine Algebras and Related Topics (Raleigh, NC, 1998). Contemporary Mathematics, vol. 248, pp. 243-291. American Mathematical Society, Providence, RI (1999)

9. Hong, J., Kang, S.J.: Introduction to Quantum Groups and Crystal Bases. Graduate Studies in Mathematics, vol. 42. American Mathematical Society, Providence, RI (2000)

10. Kashiwara, M.: Similarity of crystal bases, Lie algebras and their representations. (Seoul, 1995). Contemporary Mathematics, vol. 194, pp. 177-186. American Mathematical Society, Providence, RI (1996)

11. Kashiwara, M.: On crystal bases of the $q$-analogue of universal enveloping algebras. Duke Math. J. 63, 465-516 (1991)

12. Kashiwara, M., Nakashima, T.: Crystal graphs for representations of the $q$-analogue of classical Lie algebras. J. Algebra 165, 295-345 (1994)

13. Kirillov, A., Reshetikhin, N.: Representations of Yangians and multiplicities of the inclusion of the irreducible components of the tensor product of representations of simple Lie algebras. J. Sov. Math. 52, 3156-3164 (1990)

14. Lascoux, A., Schützenberger, M.-P.: Sur une conjecture de H. O. Foulkes. C. R. Acad. Sci. Paris Sér. I Math. 288, 95-98 (1979)

15. Lenart, C.: On the combinatorics of crystal graphs. I. Lusztig's involution. Adv. Math. 211, 324-340 (2007)

16. Lenart, C.: Haglund-Haiman-Loehr type formulas for Hall-Littlewood polynomials of type $B$ and $C$. Algebra Number Theory 4, 887-917 (2010)

17. Lenart, C.: Hall-Littlewood polynomials, alcove walks and fillings of Young diagrams. Discret. Math. 311, 258-275 (2011)

18. Lenart, C.: From Macdonald polynomials to a charge statistic beyond type A. J. Combin. Theory Ser. A 119, 683-712 (2012)

19. Lenart, C., Lubovsky, A.: A uniform realization of the combinatorial $R$-matrix. In preparation

20. Lenart, C., Naito, S., Sagaki, D., Schilling, A., Shimozono, M.: Explicit description of the action of root operators on quantum Lakshmibai-Seshadri paths. arXiv:1308.3529. To appear in Proceedings of the 5th Mathematical Society of Japan Seasonal Institute. Schubert Calculus, Osaka, Japan (2012)

21. Lenart, C., Naito, S., Sagaki, D., Schilling, A., Shimozono, M.: A uniform model for KirillovReshetikhin crystals I: Lifting the parabolic quantum Bruhat graph. Int. Math. Res. Not. (2014). doi:10. 1093/imrn/rnt263

22. Lenart, C., Naito, S., Sagaki, D., Schilling, A., Shimozono, M.: A uniform model for KirillovReshetikhin crystals II: Alcove model, path models and $P=X$. arXiv:1402.2203. Extended abstract in 
25th International Conference on Formal Power Series and Algebraic Combinatorics (FPSAC 2013), Discret. Math. Theor. Comput. Sci. Proc. AS, pp. 57-68, Paris, France (2013)

23. Lenart, C., Postnikov, A.: Affine Weyl groups in $K$-theory and representation theory. Int. Math. Res. Not., pp. 1-65. Art. ID rnm038 (2007)

24. Lenart, C., Postnikov, A.: A combinatorial model for crystals of Kac-Moody algebras. Trans. Am. Math. Soc. 360, 4349-4381 (2008)

25. Lenart, C., Schilling, A.: Crystal energy functions via the charge in types $A$ and $C$. Math. Z. 273, 401-426 (2013)

26. Littelmann, P.: A Littlewood-Richardson rule for symmetrizable Kac-Moody algebras. Invent. Math. 116, 329-346 (1994)

27. Littelmann, P.: Paths and root operators in representation theory. Ann. Math. 142, 499-525 (1995)

28. Naito, S., Sagaki, D.: Lakshmibai-Seshadri paths of level-zero weight shape and one-dimensional sums associated to level-zero fundamental representations. Compos. Math. 144, 1525-1556 (2008)

29. Nakayashiki, A., Yamada, Y.: Kostka polynomials and energy functions in solvable lattice models. Selecta Math. (N.S.) 3, 547-599 (1997)

30. Ram, A., Yip, M.: A combinatorial formula for Macdonald polynomials. Adv. Math. 226, 309-331 (2011)

31. Sheats, J.T.: A symplectic jeu de taquin bijection between the tableaux of King and of De Concini. Trans. Am. Math. Soc. 351, 3569-3607 (1999)

32. Schilling, A., Tingley, P.: Demazure crystals, Kirillov-Reshetikhin crystals, and the energy function. Electron. J. Combin. (2012)

33. Yamane, Y.: Perfect crystals of $U_{q}\left(G_{2}^{(1)}\right)$. J. Algebra 210, 440-486 (1998) 